Pure and Applied Mathematics Quarterly

Volume 7, Number 4

(Special Issue:

In memory of Eckart Viehweg)

1165-1207, 2011

\title{
Holomorphic Morse Inequalities and The Green-Griffiths-Lang Conjecture
}

\author{
Jean-Pierre Demailly \\ Dedicated to the memory of Eckart Viehweg
}

\begin{abstract}
The goal of this work is to study the existence and properties of non entire curves $f: \mathbb{C} \rightarrow X$ drawn in a complex irreducible $n$-dimensional variety $X$, and more specifically to show that they must satisfy certain global algebraic or differential equations as soon as $X$ is projective of general type. By means of holomorphic Morse inequalities and a probabilistic analysis of the cohomology of jet spaces, we are able to prove a significant step of a generalized version of the Green-Griffiths-Lang conjecture on the algebraic degeneracy of entire curves.
\end{abstract}

Résumé. Le but de ce travail est d'étudier l'existence et les propriétés des courbes entières $f: \mathbb{C} \rightarrow X$ tracées sur une varété complexe irréductible de dimension $n$, et plus précisément de montrer que ces courbes doivent satisfaire à certaines équations algébriques ou différentielles globales dès que $X$ est projective de type général. Au moyen des inégalités de Morse holomorphes et d'une analyse probabiliste de la cohomologie des espaces de jets, nous démontrons une première étape significative d'une version généralisée de la conjecture de Green-GriffithsLang sur la dégénerescence algébrique des courbes entières.

Keywords: Chern curvature, holomorphic Morse inequality, jet bundle, cohomology group, entire curve, algebraic degeneration, weighted projective space, Green-Griffiths-Lang conjecture.

Received: Nov. 15, 2010; Revised: Nov. 28, 2010

MSC 2010 Classification. 32Q45, 32L20, 14C30 
Mots-clés. Courbure de Chern, inégalité de Morse holomorphe, fibré de jets, groupe de cohomologie, courbe entière, dégénérescence algébrique, espace projectif à poids, conjecture de Green-Griffiths-Lang.

\section{INTRODUCTION}

Let $X$ be a complex $n$-dimensional manifold ; most of the time we will assume that $X$ is compact and even projective algebraic. By an "entire curve" we always mean a non constant holomorphic map defined on the whole complex line $\mathbb{C}$, and we say that it is algebraically degenerate if its image is contained in a proper algebraic subvariety of the ambient variety. If $\mu: \tilde{X} \rightarrow X$ is a modification and $f: \mathbb{C} \rightarrow X$ is an entire curve whose image $f(\mathbb{C})$ is not contained in the image $\mu(E)$ of the exceptional locus, then $f$ admits a unique lifting $\widetilde{f}: \mathbb{C} \rightarrow \widetilde{X}$. For this reason, the study of the algebraic degeneration of $f$ is a birationally invariant problem, and singularities do not play an essential role at this stage. We will therefore assume that $X$ is non singular, possibly after performing a suitable composition of blow-ups. We are interested more generally in the situation where the tangent bundle $T_{X}$ is equipped with a linear subspace $V \subset T_{X}$, that is, an irreducible complex analytic subset of the total space of $T_{X}$ such that

$$
\text { all fibers } V_{x}:=V \cap T_{X, x} \text { are vector subspaces of } T_{X, x} \text {. }
$$

Then the problem is to study entire curves $f: \mathbb{C} \rightarrow X$ which are tangent to $V$, i.e. such that $f_{*} T_{\mathbb{C}} \subset V$. We will refer to a pair $(X, V)$ as being a directed variety (or directed manifold). A morphism of directed varieties $\Phi:(X, V) \rightarrow(Y, W)$ is a holomorphic map $\Phi: X \rightarrow Y$ such that $\Phi_{*} V \subset W$; by the irreducibility, it is enough to check this condition over the dense open subset $X \backslash \operatorname{Sing}(V)$ where $V$ is actually a subbundle. Here $\operatorname{Sing}(V)$ denotes the indeterminacy set of the associated meromorphic map $\alpha: X \rightarrow G_{r}\left(T_{X}\right)$ to the Grassmannian bbundle of $r$-planes in $T_{X}, r=\operatorname{rank} V$; we thus have $V_{\mid X \backslash \operatorname{Sing}(V)}=\alpha^{*} S$ where $S \rightarrow G_{r}\left(T_{X}\right)$ is the tautological subbundle of $G_{r}\left(T_{X}\right)$. In that way, we get a category, and we will be mostly interested in the subcategory whose objects $(X, V)$ are projective algebraic manifolds equipped with algebraic linear subspaces. Notice that an entire curve $f: \mathbb{C} \rightarrow X$ tangent to $V$ is just a morphism $f:\left(\mathbb{C}, T_{\mathbb{C}}\right) \rightarrow(X, V)$.

The case where $V=T_{X / S}$ is the relative tangent space of some fibration $X \rightarrow S$ is of special interest, and so is the case of a foliated variety (this is the 
situation where the sheaf of sections $\mathcal{O}(V)$ satisfies the Frobenius integrability condition $[\mathcal{O}(V), \mathcal{O}(V)] \subset \mathcal{O}(V))$; however, it is very useful to allow as well non integrable linear subspaces $V$. We refer to $V=T_{X}$ as being the absolute case. Our main target is the following deep conjecture concerning the algebraic degeneracy of entire curves, which generalizes similar statements made in [GG79] (see also [Lang86, Lang87]).

(0.2) Generalized Green-Griffiths-Lang conjecture. Let $(X, V)$ be a projective directed manifold such that the canonical sheaf $K_{V}$ is big (in the absolute case $V=T_{X}$, this means that $X$ is a variety of general type, and in the relative case we will say that $(X, V)$ is of general type). Then there should exist an algebraic subvariety $Y \subsetneq X$ such that every non constant entire curve $f: \mathbb{C} \rightarrow X$ tangent to $V$ is contained in $Y$.

The precise meaning of $K_{V}$ and of its bigness will be explained below - our definition does not coincide with other frequently used definitions and is in our view better suited to the study of entire curves of $(X, V)$. One says that $(X, V)$ is Brody-hyperbolic when there are no entire curves tangent to $V$. According to (generalized versions of) conjectures of Kobayashi [Kob70, Kob76] the hyperbolicity of $(X, V)$ should imply that $K_{V}$ is big, and even possibly ample, in a suitable sense. It would then follow from conjecture $(0.2)$ that $(X, V)$ is hyperbolic if and only if for every irreducible variety $Y \subset X$, the linear subspace $V_{\widetilde{Y}}=\overline{T_{\widetilde{Y} \backslash E} \cap \mu_{*}^{-1} V} \subset T_{\widetilde{Y}}$ has a big canonical sheaf whenever $\mu: \widetilde{Y} \rightarrow Y$ is a desingularization and $E$ is the exceptional locus.

The most striking fact known at this date on the Green-Griffiths-Lang conjecture is a recent result of Diverio, Merker and Rousseau [DMR10] in the absolute case, confirming the statement when $X \subset \mathbb{P}_{\mathbb{C}}^{n+1}$ is a generic non singular hypersurface of large degree $d$, with a (non optimal) sufficient lower bound $d \geq 2^{n^{5}}$. Their proof is based in an essential way on a strategy developed by Siu [Siu02, Siu04], combined with techniques of [Dem95]. Notice that if the Green-GriffithsLang conjecture holds true, a much stronger and probably optimal result would be true, namely all smooth hypersurfaces of degree $d \geq n+3$ would satisfy the expected algebraic degeneracy statement. Moreover, by results of Clemens [Cle86] and Voisin [Voi96], a (very) generic hypersurface of degree $d \geq 2 n+1$ would in fact be hyperbolic for every $n \geq 2$. Such a generic hyperbolicity statement has been obtained unconditionally by McQuillan [McQ98, McQ99] when $n=2$ and 
$d \geq 35$, and by Demailly-El Goul [DEG00] when $n=2$ and $d \geq 21$. Recently Diverio-Trapani [DT10] proved the same result when $n=3$ and $d \geq 593$. By definition, proving the algebraic degeneracy means finding a non zero polynomial $P$ on $X$ such that all entire curves $f: \mathbb{C} \rightarrow X$ satisfy $P(f)=0$. All known methods of proof are based on establishing first the existence of certain algebraic differential equations $P\left(f ; f^{\prime}, f^{\prime \prime}, \ldots, f^{(k)}\right)=0$ of some order $k$, and then trying to find enough such equations so that they cut out a proper algebraic locus $Y \subsetneq X$.

Let $J_{k} V$ be the space of $k$-jets of curves $f:(\mathbb{C}, 0) \rightarrow X$ tangent to $V$. One defines the sheaf $\mathcal{O}\left(E_{k, m}^{\mathrm{GG}} V^{*}\right)$ of jet differentials of order $k$ and degree $m$ to be the sheaf of holomorphic functions $P\left(z ; \xi_{1}, \ldots \xi_{k}\right)$ on $J_{k} V$ which are homogeneous polynomials of degree $m$ on the fibers of $J^{k} V \rightarrow X$ with respect to local coordinate derivatives $\xi_{j}=f^{(j)}(0)$ (see below in case $V$ has singularities). The degree $m$ considered here is the weighted degree with respect to the natural $\mathbb{C}^{*}$ action on $J^{k} V$ defined by $\lambda \cdot f(t):=f(\lambda t)$, i.e. by reparametrizing the curve with a homothetic change of variable. Since $(\lambda \cdot f)^{(j)}(t)=\lambda^{j} f^{(j)}(\lambda t)$, the weighted action is given in coordinates by

$$
\lambda \cdot\left(\xi_{1}, \xi_{2}, \ldots, \xi_{k}\right)=\left(\lambda \xi_{1}, \lambda^{2} \xi_{2}, \ldots, \lambda^{k} \xi_{k}\right)
$$

One of the major tool of the theory is the following result due to Green-Griffiths [GG79] (see also [Blo26], [Dem95, Dem97], [SY96a, SY96b], [Siu97]).

(0.4) Fundamental vanishing theorem. Let $(X, V)$ be a directed projective variety and $f:\left(\mathbb{C}, T_{\mathbb{C}}\right) \rightarrow(X, V)$ an entire curve tangent to $V$. Then for every global section $P \in H^{0}\left(X, E_{k, m}^{\mathrm{GG}} V^{*} \otimes \mathcal{O}(-A)\right)$ where $A$ is an ample divisor of $X$, one has $P\left(f ; f^{\prime}, f^{\prime \prime}, \ldots, f^{(k)}\right)=0$.

It is expected that the global sections of $H^{0}\left(X, E_{k, m}^{\mathrm{GG}} V^{*} \otimes \mathcal{O}(-A)\right)$ are precisely those which ultimately define the algebraic locus $Y \subsetneq X$ where the curve $f$ should lie. The problem is then reduced to the question of showing that there are many non zero sections of $H^{0}\left(X, E_{k, m}^{\mathrm{GG}} V^{*} \otimes \mathcal{O}(-A)\right)$, and further, understanding what is their joint base locus. The first part of this program is the main result of the present paper. 
(0.5) Theorem. Let $(X, V)$ be a directed projective variety such that $K_{V}$ is big and let $A$ be an ample divisor. Then for $k \gg 1$ and $\delta \in \mathbb{Q}_{+}$small enough, $\delta \leq c(\log k) / k$, the number of sections $h^{0}\left(X, E_{k, m}^{\mathrm{GG}} V^{*} \otimes \mathcal{O}(-m \delta A)\right)$ has maximal growth, i.e. is larger that $c_{k} m^{n+k r-1}$ for some $m \geq m_{k}$, where $c, c_{k}>0$, $n=\operatorname{dim} X$ and $r=\operatorname{rank} V$. In particular, entire curves $f:\left(\mathbb{C}, T_{\mathbb{C}}\right) \rightarrow(X, V)$ satisfy (many) algebraic differential equations.

The statement is very elementary to check when $r=\operatorname{rank} V=1$, and therefore when $n=\operatorname{dim} X=1$. In higher dimensions $n \geq 2$, only very partial results were known at this point, concerning merely the absolute case $V=T_{X}$. In dimension 2, Theorem 0.5 is a consequence of the Riemann-Roch calculation of Green-Griffiths [GG79], combined with a vanishing theorem due to Bogomolov [Bog79] - the latter actually only applies to the top cohomology group $H^{n}$, and things become much more delicate when extimates of intermediate cohomology groups are needed. In higher dimensions, Diverio [Div09] proved the existence of sections of $H^{0}\left(X, E_{k, m}^{\mathrm{GG}} V^{*} \otimes \mathcal{O}(-1)\right)$ whenever $X$ is a hypersurface of $\mathbb{P}_{\mathbb{C}}^{n+1}$ of high degree $d \geq d_{n}$, assuming $k \geq n$ and $m \geq m_{n}$. More recently, Merker [Mer10] was able to treat the case of arbitrary hypersurfaces of general type, i.e. $d \geq n+3$, assuming this time $k$ to be very large. The latter result is obtained through explicit algebraic calculations of the spaces of sections, and the proof is computationally very intensive. Bérczi [Ber10] also obtained related results with a different approach based on residue formulas, assuming $d \geq 2^{7 n \log n}$.

All these approaches are algebraic in nature, and while they use some form of holomorphic Morse inequalities [Dem85], they only require a very special elementary algebraic case, namely the lower bound

$$
h^{0}\left(X, L^{\otimes m}\right) \geq \frac{m^{n}}{n !}\left(A^{n}-n A^{n-1} \cdot B\right)-o\left(m^{n}\right)
$$

for $L=\mathcal{O}(A-B)$ with $A, B$ nef (cf. Trapani [Tra95]). Here, our techniques are based on more elaborate curvature estimates in the spirit of Cowen-Griffiths [CG76]. They require the stronger analytic form of holomorphic Morse inequalities.

(0.6) Holomorphic Morse inequalities ([Dem85]). Let $(L, h)$ be a holomorphic line bundle on a compact complex manifold $X$, equipped with a smooth hermitian metric $h$, and let $E$ be a holomorphic vector bundle. Denote by 
$\Theta_{L, h}=-\frac{i}{2 \pi} \partial \bar{\partial} \log h$ the curvature form of $(L, h)$ and consider the open set $X(L, h, q)=\left\{x \in X ; \Theta_{L, h}(x)\right.$ has signature $\left.(n-q, q)\right\}\left(q-\right.$ index set of $\left.\Theta_{L, h}\right)$, so that there is a partition $X=S \cup \bigcup_{0 \leq q \leq n} X(L, h, q)$ where $S=\left\{\operatorname{det} \Theta_{L, h}(x)=0\right\}$. Then, if we put $r=\operatorname{rank} E$, we have asymptotically as $m$ tends to infinity:

(a) (Weak Morse inequalities)

$$
h^{q}\left(X, E \otimes L^{\otimes m}\right) \leq \frac{m^{n}}{n !} r \int_{X(L, h, q)}(-1)^{q} \Theta_{L, h}^{n}+o\left(m^{n}\right) .
$$

(b) (Strong Morse inequalities) If $X(L, h, \leq q)=\coprod_{j \leq q} X(L, h, j)$, then

$$
\sum_{j=0}^{q}(-1)^{q-j} h^{j}\left(X, E \otimes L^{\otimes m}\right) \leq \frac{m^{n}}{n !} r \int_{X(L, h, \leq q)}(-1)^{q} \Theta_{L, h}^{n}+o\left(m^{n}\right) .
$$

(c) (Lower bound on $h^{0}$ )

$$
h^{0}\left(X, E \otimes L^{\otimes m}\right)-h^{1}\left(X, E \otimes L^{\otimes m}\right) \geq \frac{m^{n}}{n !} r \int_{X(L, h, \leq 1)} \Theta_{L, h}^{n}-o\left(m^{n}\right) .
$$

The proof of the above is based on refined spectral estimates for the complex Laplace-Beltrami operators. Observe that $(0.6 \mathrm{c})$ is just the special case of $(0.6 \mathrm{~b})$ when $q=1$. It has been recently observed that these inequalities should be optimal in the sense that the asymptotic cohomology functional $\widehat{h}^{q}(X, L):=$ $\lim \sup _{m \rightarrow+\infty} \frac{n !}{m^{n}} h^{0}\left(X, L^{\otimes m}\right)$ satisfies

$$
\widehat{h}^{q}(X, L) \leq \inf _{h \in C^{\infty}} \int_{X(L, h, q)}(-1)^{q} \Theta_{L, h}^{n},
$$

and that conjecturally the inequality should be an equality; it is proved in [Dem10a], [Dem10b] that this is indeed the case if $n \leq 2$ or $q=0$, at least when $X$ is projective algebraic.

Notice that holomorphic Morse inequalities are essentially insensitive to singularities, as we can pass to non singular models and blow-up $X$ as much as we want: if $\mu: \widetilde{X} \rightarrow X$ is a modification then $\mu_{*} \mathcal{O}_{\widetilde{X}}=\mathcal{O}_{X}$ and $R^{q} \mu_{*} \mathcal{O}_{\widetilde{X}}$ is supported on a codimension 1 analytic subset (even codimension 2 if $X$ is smooth). It follows by the Leray spectral sequence that the estimates for $L$ on $X$ or for $\widetilde{L}=\mu^{*} L$ on $\widetilde{X}$ differ by negligible terms $O\left(m^{n-1}\right)$. Finally, we can even work with singular hermitian metrics $h$ which have analytic singularities with positive 
rational coefficients, that is, one can write locally $h=e^{-\varphi}$ where, possibly after blowing up,

$$
\varphi(z)=c \log \sum_{j}\left|g_{j}\right|^{2} \bmod C^{\infty}, \text { with } c \in \mathbb{Q}_{+} \text {and } g_{j} \text { holomorphic } .
$$

Especially, $\varphi$ is smooth on some Zariski open set $X \backslash Z$ where $Z=\bigcap g_{j}^{-1}(0)$, and it has logarithmic poles along $Z$. Blowing-up the ideal sheaf $\mathcal{J}=\left(g_{j}\right)$ leads to divisorial singularities, and then by replacing $L$ with $\widetilde{L}=\mu^{*} L \otimes \mathcal{O}(-E)$ where $E \in$ $\operatorname{Div}_{\mathbb{Q}}(\widetilde{X})$ is the singularity divisor, we see that holomorphic Morse inequalities still hold for the sequence of groups $H^{q}\left(X, E \otimes L^{\otimes m} \otimes \mathcal{I}\left(h^{\otimes m}\right)\right)$ where $\mathcal{I}\left(h^{\otimes m}\right)$ is the multiplier ideal sheaf of $h^{\otimes m}$ (see Bonavero [Bon93] for more details). In the case of linear subspaces $V \subset T_{X}$, we introduce singular hermitian metrics as follows.

(0.9) Definition. A singular hermitian metric on a linear subspace $V \subset T_{X}$ is a metric $h$ on the fibers of $V$ such that the function $\log h: \xi \mapsto \log |\xi|_{h}^{2}$ is locally integrable on the total space of $V$.

Such a metric can also be viewed as a singular hermitian metric on the tautological line bundle $\mathcal{O}_{P(V)}(-1)$ on the projectivized bundle $P(V)=V \backslash\{0\} / \mathbb{C}^{*}$, and therefore its dual metric $h^{*}$ defines a curvature current $\Theta_{\mathcal{O}_{P(V)}(1), h^{*}}$ of type $(1,1)$ on $P(V) \subset P\left(T_{X}\right)$, such that

$$
p^{*} \Theta_{\mathcal{O}_{P(V)}(1), h^{*}}=\frac{i}{2 \pi} \partial \bar{\partial} \log h, \quad \text { where } p: V \backslash\{0\} \rightarrow P(V) .
$$

If $\log h$ is quasi-plurisubharmonic (or quasi-psh, which means psh modulo addition of a smooth function) on $V$, then $\log h$ is indeed locally integrable, and we have moreover

$$
\Theta_{\mathcal{O}_{P(V)}(1), h^{*}} \geq-C \omega
$$

for some smooth positive $(1,1)$-form on $P(V)$ and some constant $C>0$; conversely, if (0.10) holds, then $\log h$ is quasi psh.

(0.11) Definition. We will say that a singular hermitian metric $h$ on $V$ is admissible if $h$ can be written as $h=e^{\varphi} h_{0 \mid V}$ where $h_{0}$ is a smooth positive definite hermitian on $T_{X}$ and $\varphi$ is a quasi-psh weight with analytic singularities on $X$, as in (0.9). Then $h$ can be seen as a singular hermitian metric on $\mathcal{O}_{P(V)}(1)$, with the property that it induces a smooth positive definite metric on a Zariski open 
set $X^{\prime} \subset X \backslash \operatorname{Sing}(V)$; we will denote by $\operatorname{Sing}(h) \supset \operatorname{Sing}(V)$ the complement of the largest such Zariski open set $X^{\prime}$.

If $h$ is an admissible metric, we define $\mathcal{O}_{h}\left(V^{*}\right)$ to be the sheaf of germs of holomorphic sections sections of $V_{\mid X \backslash \operatorname{Sing}(h)}^{*}$ which are $h^{*}$-bounded near $\operatorname{Sing}(h)$; by the assumption on the analytic singularities, this is a coherent sheaf (as the direct image of some coherent sheaf on $P(V)$ ), and actually, since $h^{*}=e^{-\varphi} h_{0}^{*}$, it is a subsheaf of the sheaf $\mathcal{O}\left(V^{*}\right):=\mathcal{O}_{h_{0}}\left(V^{*}\right)$ associated with a smooth positive definite metric $h_{0}$ on $T_{X}$. If $r$ is the generic rank of $V$ and $m$ a positive integer, we define similarly $K_{V, h}^{m}$ to be sheaf of germs of holomorphic sections of $\left(\operatorname{det} V_{\mid X^{\prime}}^{*}\right)^{\otimes m}=\left(\Lambda^{r} V_{\mid X^{\prime}}^{*}\right)^{\otimes m}$ which are $\operatorname{det} h^{*}$-bounded, and $K_{V}^{m}:=K_{V, h_{0}}^{m}$.

If $V$ is defined by $\alpha: X \rightarrow G_{r}\left(T_{X}\right)$, there always exists a modification $\mu$ : $\widetilde{X} \rightarrow X$ such that the composition $\alpha \circ \mu: \widetilde{X} \rightarrow G_{r}\left(\mu^{*} T_{X}\right)$ becomes holomorphic, and then $\mu^{*} V_{\mid \mu^{-1}(X \backslash \operatorname{Sing}(V))}$ extends as a locally trivial subbundle of $\mu^{*} T_{X}$ which we will simply denote by $\mu^{*} V$. If $h$ is an admissible metric on $V$, then $\mu^{*} V$ can be equipped with the metric $\mu^{*} h=e^{\varphi \circ \mu} \mu^{*} h_{0}$ where $\mu^{*} h_{0}$ is smooth and positive definite. We may assume that $\varphi \circ \mu$ has divisorial singularities (otherwise just perform further blow-ups of $\widetilde{X}$ to achieve this). We then see that there is an integer $m_{0}$ such that for all multiples $m=p m_{0}$ the pull-back $\mu^{*} K_{V, h}^{m}$ is an invertible sheaf on $\tilde{X}$, and $\operatorname{det} h^{*}$ induces a smooth non singular metric on it (when $h=h_{0}$, we can even take $m_{0}=1$ ). By definition we always have $K_{V, h}^{m}=$ $\mu_{*}\left(\mu^{*} K_{V, h}^{m}\right)$ for any $m \geq 0$. In the sequel, however, we think of $K_{V, h}$ not really as a coherent sheaf, but rather as the "virtual" $\mathbb{Q}$-line bundle $\mu_{*}\left(\mu^{*} K_{V, h}^{m_{0}}\right)^{1 / m_{0}}$, and we say that $K_{V, h}$ is big if $h^{0}\left(X, K_{V, h}^{m}\right) \geq c m^{n}$ for $m \geq m_{1}$, with $c>0$, i.e. if the invertible sheaf $\mu^{*} K_{V, h}^{m_{0}}$ is big in the usual sense.

At this point, it is important to observe that "our" canonical sheaf $K_{V}$ differs from the sheaf $\mathcal{K}_{V}:=i_{*} \mathcal{O}\left(K_{V}\right)$ associated with the injection $i: X \backslash \operatorname{Sing}(V) \hookrightarrow X$, which is usually referred to as being the "canonical sheaf", at least when $V$ is the space of tangents to a foliation. In fact, $\mathcal{K}_{V}$ is always an invertible sheaf and there is an obvious inclusion $K_{V} \subset \mathcal{K}_{V}$. More precisely, the image of $\mathcal{O}\left(\Lambda^{r} T_{X}^{*}\right) \rightarrow \mathcal{K}_{V}$ is equal to $\mathcal{K}_{V} \otimes_{\mathcal{O}_{X}} \mathcal{J}$ for a certain coherent ideal $\mathcal{J} \subset \mathcal{O}_{X}$, and the condition to have $h_{0}$-bounded sections on $X \backslash \operatorname{Sing}(V)$ precisely means that our sections are bounded by Const $\sum\left|g_{j}\right|$ in terms of the generators $\left(g_{j}\right)$ of $\mathcal{K}_{V} \otimes_{\mathcal{O}_{X}} \mathcal{J}$, i.e. $K_{V}=\mathcal{K}_{V} \otimes_{\mathcal{O}_{X}} \overline{\mathcal{J}}$ where $\overline{\mathcal{J}}$ is the integral closure of $\mathcal{J}$. More generally,

$$
K_{V, h}^{m}=\mathcal{K}_{V}^{m} \otimes_{\mathcal{O}_{X}} \overline{\mathcal{J}}_{h, m_{0}}^{m / m_{0}}
$$


where $\overline{\mathcal{J}}_{h, m_{0}}^{m / m_{0}} \subset \mathcal{O}_{X}$ is the $\left(m / m_{0}\right)$-integral closure of a certain ideal sheaf $\mathcal{J}_{h, m_{0}} \subset \mathcal{O}_{X}$, which can itself be assumed to be integrally closed; in our previous discussion, $\mu$ is chosen so that $\mu^{*} \mathcal{J}_{h, m_{0}}$ is invertible on $\widetilde{X}$.

The discrepancy already occurs e.g. with the rank 1 linear space $V \subset T_{\mathbb{P}_{\mathbb{C}}^{n}}$ consisting at each point $z \neq 0$ of the tangent to the line $(0 z)$ (so that necessarily $\left.V_{0}=T_{\mathbb{P}_{\mathbb{C}}^{n}, 0}\right)$. As a sheaf (and not as a linear space), $i_{*} \mathcal{O}(V)$ is the invertible sheaf generated by the vector field $\xi=\sum z_{j} \partial / \partial z_{j}$ on the affine open set $\mathbb{C}^{n} \subset \mathbb{P}_{\mathbb{C}}^{n}$, and therefore $\mathcal{K}_{V}:=i_{*} \mathcal{O}\left(V^{*}\right)$ is generated over $\mathbb{C}^{n}$ by the unique 1-form $u$ such that $u(\xi)=1$. Since $\xi$ vanishes at 0 , the generator $u$ is unbounded with respect to a smooth metric $h_{0}$ on $T_{\mathbb{P}_{\mathbb{C}}^{n}}^{n}$, and it is easily seen that $K_{V}$ is the non invertible sheaf $K_{V}=\mathcal{K}_{V} \otimes \mathfrak{m}_{\mathbb{P}_{\mathbb{C}}^{n}, 0}$. We can make it invertible by considering the blow-up $\mu: \widetilde{X} \rightarrow X$ of $X=\mathbb{P}_{\mathbb{C}}^{n}$ at 0 , so that $\mu^{*} K_{V}$ is isomorphic to $\mu^{*} \mathcal{K}_{V} \otimes \mathcal{O}_{\widetilde{X}}(-E)$ where $E$ is the exceptional divisor. The integral curves $C$ of $V$ are of course lines through 0 , and when a standard parametrization is used, their derivatives do not vanish at 0 , while the sections of $i_{*} \mathcal{O}(V)$ do - another sign that $i_{*} \mathcal{O}(V)$ and $i_{*} \mathcal{O}\left(V^{*}\right)$ are the wrong objects to consider. Another standard example is obtained by taking a generic pencil of elliptic curves $\lambda P(z)+\mu Q(z)=0$ of degree 3 in $\mathbb{P}_{\mathbb{C}}^{2}$, and the linear space $V$ consisting of the tangents to the fibers of the rational map $\mathbb{P}_{\mathbb{C}}^{2}--\rightarrow \mathbb{P}_{\mathbb{C}}^{1}$ defined by $z \mapsto Q(z) / P(z)$. Then $V$ is given by

$$
0 \longrightarrow i_{*} \mathcal{O}(V) \longrightarrow \mathcal{O}\left(T_{\mathbb{P}_{\mathbb{C}}^{2}} \stackrel{P d Q-Q d P}{\longrightarrow} \mathcal{O}_{\mathbb{P}_{\mathbb{C}}^{2}}(6) \otimes \mathcal{J}_{S} \longrightarrow 0\right.
$$

where $S=\operatorname{Sing}(V)$ consists of the 9 points $\{P(z)=0\} \cap\{Q(z)=0\}$, and $\mathcal{J}_{S}$ is the corresponding ideal sheaf of $S$. Since $\operatorname{det} \mathcal{O}\left(T_{\mathbb{P}^{2}}\right)=\mathcal{O}(3)$, we see that $\mathcal{K}_{V}=\mathcal{O}(3)$ is ample, which seems to contradict (0.2) since all leaves are elliptic curves. There is however no such contradiction, because $K_{V}=\mathcal{K}_{V} \otimes \mathcal{J}_{S}$ is not big in our sense (it has degree 0 on all members of the elliptic pencil). A similar example is obtained with a generic pencil of conics, in which case $\mathcal{K}_{V}=\mathcal{O}(1)$ and $\operatorname{card} S=4$.

For a given admissible hermitian structure $(V, h)$, we define similarly the sheaf $E_{k, m}^{\mathrm{GG}} V_{h}^{*}$ to be the sheaf of polynomials defined over $X \backslash \operatorname{Sing}(h)$ which are " $h$-bounded". This means that when they are viewed as polynomials $P\left(z ; \xi_{1}, \ldots, \xi_{k}\right)$ in terms of $\xi_{j}=\left(\nabla_{h_{0}}^{1,0}\right)^{j} f(0)$ where $\nabla_{h_{0}}^{1,0}$ is the $(1,0)$-component of the induced Chern connection on $\left(V, h_{0}\right)$, there is a uniform bound

$$
\left|P\left(z ; \xi_{1}, \ldots, \xi_{k}\right)\right| \leq C\left(\sum\left\|\xi_{j}\right\|_{h}^{1 / j}\right)^{m}
$$


near points of $X \backslash X^{\prime}$ (see section 2 for more details on this). Again, by a direct image argument, one sees that $E_{k, m}^{\mathrm{GG}} V_{h}^{*}$ is always a coherent sheaf. The sheaf $E_{k, m}^{\mathrm{GG}} V^{*}$ is defined to be $E_{k, m}^{\mathrm{GG}} V_{h}^{*}$ when $h=h_{0}$ (it is actually independent of the choice of $h_{0}$, as follows from arguments similar to those given in section 2). Notice that this is exactly what is needed to extend the proof of the vanishing theorem 0.4 to the case of a singular linear space $V$; the value distribution theory argument can only work when the functions $P\left(f ; f^{\prime}, \ldots, f^{(k)}\right)(t)$ do not exhibit poles, and this is guaranteed here by the boundedness assumption.

Our strategy can be described as follows. We consider the Green-Griffiths bundle of $k$-jets $X_{k}^{\mathrm{GG}}=J^{k} V \backslash\{0\} / \mathbb{C}^{*}$, which by (0.3) consists of a fibration in weighted projective spaces, and its associated tautological sheaf

$$
L=\mathcal{O}_{X_{k}^{\mathrm{GG}}}(1),
$$

viewed rather as a virtual $\mathbb{Q}$-line bundle $\mathcal{O}_{X_{k}^{\mathrm{GG}}}\left(m_{0}\right)^{1 / m_{0}}$ with $m_{0}=\operatorname{lcm}(1,2, \ldots, k)$. Then, if $\pi_{k}: X_{k}^{\mathrm{GG}} \rightarrow X$ is the natural projection, we have

$$
E_{k, m}^{\mathrm{GG}}=\left(\pi_{k}\right)_{*} \mathcal{O}_{X_{k}^{\mathrm{GG}}}(m) \text { and } R^{q}\left(\pi_{k}\right)_{*} \mathcal{O}_{X_{k}^{\mathrm{GG}}}(m)=0 \text { for } q \geq 1 .
$$

Hence, by the Leray spectral sequence we get for every invertible sheaf $F$ on $X$ the isomorphism

$$
H^{q}\left(X, E_{k, m}^{\mathrm{GG}} V^{*} \otimes F\right) \simeq H^{q}\left(X_{k}^{\mathrm{GG}}, \mathcal{O}_{X_{k}^{\mathrm{GG}}}(m) \otimes \pi_{k}^{*} F\right)
$$

The latter group can be evaluated thanks to holomorphic Morse inequalities. In fact we can associate with any admissible metric $h$ on $V$ a metric (or rather a natural family) of metrics on $L=\mathcal{O}_{X_{k}^{\mathrm{GG}}}(1)$. The space $X_{k}^{\mathrm{GG}}$ always possesses quotient singularities if $k \geq 2$ (and even some more if $V$ is singular), but we do not really care since Morse inequalities still work in this setting. As we will see, it is then possible to get nice asymptotic formulas as $k \rightarrow+\infty$. They appear to be of a probabilistic nature if we take the components of the $k$-jet (i.e. the successive derivatives $\xi_{j}=f^{(j)}(0), 1 \leq j \leq k$ ) as random variables. This probabilistic behaviour was somehow already visible in the Riemann-Roch calculation of [GG79]. In this way, assuming $K_{V}$ big, we produce a lot of sections $\sigma_{j}=H^{0}\left(X_{k}^{\mathrm{GG}}, \mathcal{O}_{X_{k}^{\mathrm{GG}}}(m) \otimes \pi_{k}^{*} F\right)$, corresponding to certain divisors $Z_{j} \subset X_{k}^{\mathrm{GG}}$. The hard problem which is left in order to complete a proof of the generalized Green-Griffiths-Lang conjecture is to compute the base locus $Z=\bigcap Z_{j}$ and to show that $Y=\pi_{k}(Z) \subset X$ must be a proper algebraic variety. Although we 
cannot address this problem at present, we will indicate a few technical results and a couple of potential strategies in this direction.

I would like to thank Simone Diverio and Mihai Păun for several stimulating discussions, and Erwan Rousseau for convincing me to explain better the peculiarities of the definition of the canonical sheaf employed here.

\section{Hermitian geometry of Weighted projective spaces}

The goal of this section is to introduce natural Kähler metrics on weighted projective spaces, and to evaluate the corresponding volume forms. Here we put $d^{c}=\frac{i}{4 \pi}(\bar{\partial}-\partial)$ so that $d d^{c}=\frac{i}{2 \pi} \partial \bar{\partial}$. The normalization of the $d^{c}$ operator is chosen such that we have precisely $\left(d d^{c} \log |z|^{2}\right)^{n}=\delta_{0}$ for the Monge-Ampère operator in $\mathbb{C}^{n}$; also, for every holomorphic or meromorphic section $\sigma$ of a hermitian line bundle $(L, h)$ the Lelong-Poincaré can be formulated

$$
d d^{c} \log |\sigma|_{h}^{2}=\left[Z_{\sigma}\right]-\Theta_{L, h},
$$

where $\Theta_{L, h}=\frac{i}{2 \pi} D_{L, h}^{2}$ is the $(1,1)$-curvature form of $L$ and $Z_{\sigma}$ the zero divisor of $\sigma$. The closed $(1,1)$-form $\Theta_{L, h}$ is a representative of the first Chern class $c_{1}(L)$. Given a $k$-tuple of "weights" $a=\left(a_{1}, \ldots, a_{k}\right)$, i.e. of relatively prime integers $a_{s}>0$ we introduce the weighted projective space $P\left(a_{1}, \ldots, a_{k}\right)$ to be the quotient of $\mathbb{C}^{k} \backslash\{0\}$ by the corresponding weighted $\mathbb{C}^{*}$ action:

$$
P\left(a_{1}, \ldots, a_{k}\right)=\mathbb{C}^{k} \backslash\{0\} / \mathbb{C}^{*}, \quad \lambda \cdot z=\left(\lambda^{a_{1}} z_{1}, \ldots, \lambda^{a_{k}} z_{k}\right) .
$$

As is well known, this defines a toric $k$-1-dimensional algebraic variety with quotient singularities. On this variety, we introduce the possibly singular (but almost everywhere smooth and non degenerate) Kähler form $\omega_{a, p}$ defined by

$$
\pi_{a}^{*} \omega_{a, p}=d d^{c} \varphi_{a, p}, \quad \varphi_{a, p}(z)=\frac{1}{p} \log \sum_{1 \leq s \leq k}\left|z_{s}\right|^{2 p / a_{s}},
$$

where $\pi_{a}: \mathbb{C}^{k} \backslash\{0\} \rightarrow P\left(a_{1}, \ldots, a_{k}\right)$ is the canonical projection and $p>0$ is a positive constant. It is clear that $\varphi_{p, a}$ is real analytic on $\mathbb{C}^{k} \backslash\{0\}$ if $p$ is an integer and a common multiple of all weights $a_{s}$. It is at least $C^{2}$ is $p$ is real and $p \geq \max \left(a_{s}\right)$, which will be more than sufficient for our purposes (but everything would still work for any $p>0$ ). The resulting metric is in any case smooth and positive definite outside of the coordinate hyperplanes $z_{s}=0$, and these hyperplanes will not matter here since they are of capacity zero with respect to 
all currents $\left(d d^{c} \varphi_{a, p}\right)^{\ell}$. In order to evaluate the volume $\int_{P\left(a_{1}, \ldots, a_{k}\right)} \omega_{a, p}^{k-1}$, one can observe that

$$
\begin{aligned}
\int_{P\left(a_{1}, \ldots, a_{k}\right)} \omega_{a, p}^{k-1} & =\int_{z \in \mathbb{C}^{k}, \varphi_{a, p}(z)=0} \pi_{a}^{*} \omega_{a, p}^{k-1} \wedge d^{c} \varphi_{a, p} \\
& =\int_{z \in \mathbb{C}^{k}, \varphi_{a, p}(z)=0}\left(d d^{c} \varphi_{a, p}\right)^{k-1} \wedge d^{c} \varphi_{a, p} \\
& =\frac{1}{p^{k}} \int_{z \in \mathbb{C}^{k}, \varphi_{a, p}(z)<0}\left(d d^{c} e^{p \varphi_{a, p}}\right)^{k} .
\end{aligned}
$$

The first equality comes from the fact that $\left\{\varphi_{a, p}(z)=0\right\}$ is a circle bundle over $P\left(a_{1}, \ldots, a_{k}\right)$, by using the identities $\varphi_{a, p}(\lambda \cdot z)=\varphi_{a, p}(z)+\log |\lambda|^{2}$ and $\int_{|\lambda|=1} d^{c} \log |\lambda|^{2}=1$. The third equality can be seen by Stokes formula applied to the $(2 k-1)$-form

$$
\left(d d^{c} e^{p \varphi_{a, p}}\right)^{k-1} \wedge d^{c} e^{p \varphi_{a, p}}=e^{p \varphi_{a, p}}\left(d d^{c} \varphi_{a, p}\right)^{k-1} \wedge d^{c} \varphi_{a, p}
$$

on the pseudoconvex open set $\left\{z \in \mathbb{C}^{k} ; \varphi_{a, p}(z)<0\right\}$. Now, we find

$$
\begin{gathered}
\left(d d^{c} e^{p \varphi_{a, p}}\right)^{k}=\left(d d^{c} \sum_{1 \leq s \leq k}\left|z_{s}\right|^{2 p / a_{s}}\right)^{k}=\prod_{1 \leq s \leq k}\left(\frac{p}{a_{s}}\left|z_{s}\right|^{\frac{p}{a_{s}}-1}\right)\left(d d^{c}|z|^{2}\right)^{k}, \\
\int_{z \in \mathbb{C}^{k}, \varphi_{a, p}(z)<0}\left(d d^{c} e^{p \varphi_{a, p}}\right)^{k}=\prod_{1 \leq s \leq k} \frac{p}{a_{s}}=\frac{p^{k}}{a_{1} \ldots a_{k}} .
\end{gathered}
$$

In fact, (1.5) and (1.6) are clear when $p=a_{1}=\ldots=a_{k}=1$ (this is just the standard calculation of the volume of the unit ball in $\left.\mathbb{C}^{k}\right)$; the general case follows by substituting formally $z_{s} \mapsto z_{s}^{p / a_{s}}$, and using rotational invariance along with the observation that the arguments of the complex numbers $z_{s}^{p / a_{s}}$ now run in the interval $\left[0,2 \pi p / a_{s}\right.$ [ instead of $[0,2 \pi[$ (say). As a consequence of (1.4) and (1.6), we obtain the well known value

$$
\int_{P\left(a_{1}, \ldots, a_{k}\right)} \omega_{a, p}^{k-1}=\frac{1}{a_{1} \ldots a_{k}}
$$

for the volume. Notice that this is independent of $p$ (as it is obvious by Stokes theorem, since the cohomology class of $\omega_{a, p}$ does not depend on $p$ ). When $p$ tends to $+\infty$, we have $\varphi_{a, p}(z) \mapsto \varphi_{a, \infty}(z)=\log \max _{1 \leq s \leq k}\left|z_{s}\right|^{2 / a_{s}}$ and the volume form $\omega_{a, p}^{k-1}$ converges to a rotationally invariant measure supported by the image of the polycircle $\prod\left\{\left|z_{s}\right|=1\right\}$ in $P\left(a_{1}, \ldots, a_{k}\right)$. This is so because not all $\left|z_{s}\right|^{2 / a_{s}}$ are equal outside of the image of the polycircle, thus $\varphi_{a, \infty}(z)$ locally depends only on $k-1$ complex variables, and so $\omega_{a, \infty}^{k-1}=0$ there by log homogeneity. 
Our later calculations will require a slightly more general setting. Instead of looking at $\mathbb{C}^{k}$, we consider the weighted $\mathbb{C}^{*}$ action defined by

$$
\mathbb{C}^{|r|}=\mathbb{C}^{r_{1}} \times \ldots \times \mathbb{C}^{r_{k}}, \quad \lambda \cdot z=\left(\lambda^{a_{1}} z_{1}, \ldots, \lambda^{a_{k}} z_{k}\right) .
$$

Here $z_{s} \in \mathbb{C}^{r_{s}}$ for some $k$-tuple $r=\left(r_{1}, \ldots, r_{k}\right)$ and $|r|=r_{1}+\ldots+r_{k}$. This gives rise to a weighted projective space

$$
\begin{aligned}
& P\left(a_{1}^{\left[r_{1}\right]}, \ldots, a_{k}^{\left[r_{k}\right]}\right)=P\left(a_{1}, \ldots, a_{1}, \ldots, a_{k}, \ldots, a_{k}\right), \\
& \pi_{a, r}: \mathbb{C}^{r_{1}} \times \ldots \times \mathbb{C}^{r_{k}} \backslash\{0\} \longrightarrow P\left(a_{1}^{\left[r_{1}\right]}, \ldots, a_{k}^{\left[r_{k}\right]}\right)
\end{aligned}
$$

obtained by repeating $r_{s}$ times each weight $a_{s}$. On this space, we introduce the degenerate Kähler metric $\omega_{a, r, p}$ such that

$$
\pi_{a, r}^{*} \omega_{a, r, p}=d d^{c} \varphi_{a, r, p}, \quad \varphi_{a, r, p}(z)=\frac{1}{p} \log \sum_{1 \leq s \leq k}\left|z_{s}\right|^{2 p / a_{s}}
$$

where $\left|z_{s}\right|$ stands now for the standard hermitian norm $\left(\sum_{1 \leq j \leq r_{s}}\left|z_{s, j}\right|^{2}\right)^{1 / 2}$ on $\mathbb{C}^{r_{s}}$. This metric is cohomologous to the corresponding "polydisc-like" metric $\omega_{a, p}$ already defined, and therefore Stokes theorem implies

$$
\int_{P\left(a_{1}^{\left[r_{1}\right]}, \ldots, a_{k}^{\left[r_{k}\right]}\right)} \omega_{a, r, p}^{|r|-1}=\frac{1}{a_{1}^{r_{1}} \ldots a_{k}^{r_{k}}} .
$$

Since $\left(d d^{c} \log \left|z_{s}\right|^{2}\right)^{r_{s}}=0$ on $\mathbb{C}^{r_{s}} \backslash\{0\}$ by homogeneity, we conclude as before that the weak $\operatorname{limit} \lim _{p \rightarrow+\infty} \omega_{a, r, p}^{|r|-1}=\omega_{a, r, \infty}^{|r|-1}$ associated with

$$
\varphi_{a, r, \infty}(z)=\log \max _{1 \leq s \leq k}\left|z_{s}\right|^{2 / a_{s}}
$$

is a measure supported by the image of the product of unit spheres $\prod S^{2 r_{s}-1}$ in $P\left(a_{1}^{\left[r_{1}\right]}, \ldots, a_{k}^{\left[r_{k}\right]}\right)$, which is invariant under the action of $U\left(r_{1}\right) \times \ldots \times U\left(r_{k}\right)$ on $\mathbb{C}^{r_{1}} \times \ldots \times \mathbb{C}^{r_{k}}$, and thus coincides with the hermitian area measure up to a constant determined by condition (1.11). In fact, outside of the product of spheres, $\varphi_{a, r, \infty}$ locally depends only on at most $k-1$ factors and thus, for dimension reasons, the top power $\left(d d^{c} \varphi_{a, r, \infty}\right)^{|r|-1}$ must be zero there. In the next section, the following change of variable formula will be needed. For simplicity of exposition we restrict ourselves to continuous functions, but a standard density argument would easily extend the formula to all functions that are Lebesgue integrable with respect to the volume form $\omega_{a, r, p}^{|r|-1}$.

(1.13) Proposition. Let $f(z)$ be a bounded function on $P\left(a_{1}^{\left[r_{1}\right]}, \ldots, a_{k}^{\left[r_{k}\right]}\right)$ which is continuous outside of the hyperplane sections $z_{s}=0$. We also view $f$ as a 
$\mathbb{C}^{*}$-invariant continuous function on $\prod\left(\mathbb{C}^{r_{s}} \backslash\{0\}\right)$. Then

$$
\begin{aligned}
& \int_{P\left(a_{1}^{\left[r_{1}\right]}, \ldots, a_{k}^{\left[r_{k}\right]}\right)} f(z) \omega_{a, r, p}^{|r|-1} \\
& \quad=\frac{(|r|-1) !}{\prod_{s} a_{s}^{r_{s}}} \int_{(x, u) \in \Delta_{k-1} \times \prod S^{2 r_{s}-1}} f\left(x_{1}^{a_{1} / 2 p} u_{1}, \ldots, x_{k}^{a_{k} / 2 p} u_{k}\right) \prod_{1 \leq s \leq k} \frac{x_{s}^{r_{s}-1}}{\left(r_{s}-1\right) !} d x d \mu(u)
\end{aligned}
$$

where $\Delta_{k-1}$ is the $(k-1)$-simplex $\left\{x_{s} \geq 0, \sum x_{s}=1\right\}, d x=d x_{1} \wedge \ldots \wedge d x_{k-1}$ its standard measure, and where $d \mu(u)=d \mu_{1}\left(u_{1}\right) \ldots d \mu_{k}\left(u_{k}\right)$ is the rotation invariant probability measure on the product $\prod_{s} S^{2 r_{s}-1}$ of unit spheres in $\mathbb{C}^{r_{1}} \times \ldots \times \mathbb{C}^{r_{k}}$. As a consequence

$$
\lim _{p \rightarrow+\infty} \int_{P\left(a_{1}^{\left[r_{1}\right]}, \ldots, a_{k}^{\left[r_{k}\right]}\right)} f(z) \omega_{a, r, p}^{|r|-1}=\frac{1}{\prod_{s} a_{s}^{r_{s}}} \int_{\prod S^{2 r_{s}-1}} f(u) d \mu(u) .
$$

Proof. The area formula of the disc $\int_{|\lambda|<1} d d^{c}|\lambda|^{2}=1$ and a consideration of the unit disc bundle over $P\left(a_{1}^{\left[r_{1}\right]}, \ldots, a_{k}^{\left[r_{k}\right]}\right)$ imply that

$$
I_{p}:=\int_{P\left(a_{1}^{\left[r_{1}\right]}, \ldots, a_{k}^{\left[r_{k}\right]}\right)} f(z) \omega_{a, r, p}^{|r|-1}=\int_{z \in \mathbb{C}^{|r|}, \varphi_{a, r, p}(z)<0} f(z)\left(d d^{c} \varphi_{a, r, p}\right)^{|r|-1} \wedge d d^{c} e^{\varphi_{a, r, p}} .
$$

Now, a straightforward calculation on $\mathbb{C}^{|r|}$ gives

$$
\begin{aligned}
\left(d d^{c} e^{p \varphi_{a, r, p}}\right)^{|r|} & =\left(d d^{c} \sum_{1 \leq s \leq k}\left|z_{s}\right|^{2 p / a_{s}}\right)^{|r|} \\
& =\prod_{1 \leq s \leq k}\left(\frac{p}{a_{s}}\right)^{r_{s}+1}\left|z_{s}\right|^{2 r_{s}\left(p / a_{s}-1\right)}\left(d d^{c}|z|^{2}\right)^{|r|} .
\end{aligned}
$$

On the other hand, we have $\left(d d^{c}|z|^{2}\right)^{|r|}=\frac{|r| !}{r_{1} ! \ldots r_{k} !} \prod_{1 \leq s \leq k}\left(d d^{c}\left|z_{s}\right|^{2}\right)^{r_{s}}$ and

$$
\begin{aligned}
\left(d d^{c} e^{p \varphi_{a, r, p}}\right)^{|r|} & =\left(p e^{p \varphi_{a, r, p}}\left(d d^{c} \varphi_{a, r, p}+p d \varphi_{a, r, p} \wedge d^{c} \varphi_{a, r, p}\right)\right)^{|r|} \\
& =|r| p^{|r|+1} e^{|r| p \varphi_{a, r, p}}\left(d d^{c} \varphi_{a, r, p}\right)^{|r|-1} \wedge d \varphi_{a, r, p} \wedge d^{c} \varphi_{a, r, p} \\
& =|r| p^{|r|+1} e^{(|r| p-1) \varphi_{a, r, p}}\left(d d^{c} \varphi_{a, r, p}\right)^{|r|-1} \wedge d d^{c} e^{\varphi_{a, r, p}},
\end{aligned}
$$

thanks to the homogeneity relation $\left(d d^{c} \varphi_{a, r, p}\right)^{|r|}=0$. Putting everything together, we find

$$
I_{p}=\int_{z \in \mathbb{C}|r|, \varphi_{a, r, p}(z)<0} \frac{(|r|-1) ! p^{k-1} f(z)}{\left(\sum_{s}\left|z_{s}\right|^{2 p / a_{s}}\right)^{|r|-1 / p}} \prod_{s} \frac{\left(d d^{c}\left|z_{s}\right|^{2}\right)^{r_{s}}}{r_{s} ! a_{s}^{r_{s}+1}\left|z_{s}\right|^{2 r_{s}\left(1-p / a_{s}\right)}} .
$$


A standard calculation in polar coordinates with $z_{s}=\rho_{s} u_{s}, u_{s} \in S^{2 r_{s}-1}$, yields

$$
\frac{\left(d d^{c}\left|z_{s}\right|^{2}\right)^{r_{s}}}{\left|z_{s}\right|^{2 r_{s}}}=2 r_{s} \frac{d \rho_{s}}{\rho_{s}} d \mu_{s}\left(u_{s}\right)
$$

where $\mu_{s}$ is the $U\left(r_{s}\right)$-invariant probability measure on $S^{2 r_{s}-1}$. Therefore

$$
\begin{aligned}
I_{p} & =\int_{\varphi_{a, r, p}(z)<0} \frac{(|r|-1) ! p^{k-1} f\left(\rho_{1} u_{1}, \ldots, \rho_{k} u_{k}\right)}{\left(\sum_{1 \leq s \leq k} \rho_{s}^{2 p / a_{s}}\right)^{|r|-1 / p}} \prod_{s} \frac{2 \rho_{s}^{2 p r_{s} / a_{s}} \frac{d \rho_{s}}{\rho_{s}} d \mu_{s}\left(u_{s}\right)}{\left(r_{s}-1\right) ! a_{s}^{r_{s}+1}} \\
& =\int_{u_{s} \in S^{2 r_{s}-1}, \sum t_{s}<1} \frac{(|r|-1) ! p^{-1} f\left(t_{1}^{a_{1} / 2 p} u_{1}, \ldots, t_{k}^{a_{k} / 2 p} u_{k}\right)}{\left(\sum_{1 \leq s \leq k} t_{s}\right)^{|r|-1 / p}} \prod_{s} \frac{t_{s}^{r_{s}-1} d t_{s} d \mu_{s}\left(u_{s}\right)}{\left(r_{s}-1\right) ! a_{s}^{r_{s}}}
\end{aligned}
$$

by putting $t_{s}=\left|z_{s}\right|^{2 p / a_{s}}=\rho_{s}^{2 p / a_{s}}$, i.e. $\left.\left.\rho_{s}=t_{s}^{a_{s} / 2 p}, t_{s} \in\right] 0,1\right]$. We use still another change of variable $t_{s}=t x_{s}$ with $t=\sum_{1 \leq s \leq k} t_{s}$ and $\left.\left.x_{s} \in\right] 0,1\right], \sum_{1 \leq s \leq k} x_{s}=1$. Then

$$
d t_{1} \wedge \ldots \wedge d t_{k}=t^{k-1} d x d t \quad \text { where } d x=d x_{1} \wedge \ldots \wedge d x_{k-1} .
$$

The $\mathbb{C}^{*}$ invariance of $f$ shows that

$$
\begin{aligned}
I_{p} & =\int_{\substack{\left.\left.u_{s} \in S^{2 r_{s}-1} \\
\Sigma x_{s}=1, t \in\right] 0,1\right]}}(|r|-1) ! f\left(x_{1}^{a_{s} / 2 p} u_{1}, \ldots, x_{k}^{a_{k} / 2 p} u_{k}\right) \prod_{1 \leq s \leq k} \frac{x_{s}^{r_{s}-1} d \mu_{s}\left(u_{s}\right)}{\left(r_{s}-1\right) ! a_{s}^{r_{s}}} \frac{d x d t}{p t^{1-1 / p}} \\
& =\int_{\substack{u_{s} \in S^{2 r_{s}-1} \\
\Sigma x_{s}=1}}(|r|-1) ! f\left(x_{1}^{a_{s} / 2 p} u_{1}, \ldots, x_{k}^{a_{k} / 2 p} u_{k}\right) \prod_{1 \leq s \leq k} \frac{x_{s}^{r_{s}-1} d \mu_{s}\left(u_{s}\right)}{\left(r_{s}-1\right) ! a_{s}^{r_{s}}} d x .
\end{aligned}
$$

This is equivalent to the formula given in Proposition 1.13. We have $x_{s}^{2 a_{s} / p} \rightarrow 1$ as $p \rightarrow+\infty$, and by Lebesgue's bounded convergence theorem and Fubini's formula, we get

$$
\lim _{p \rightarrow+\infty} I_{p}=\frac{(|r|-1)}{\prod_{s} a_{s}^{r_{s}}} \int_{(x, u) \in \Delta_{k-1} \times \prod S^{2 r_{s}-1}} f(u) \prod_{1 \leq s \leq k} \frac{x_{s}^{r_{s}-1}}{\left(r_{s}-1\right) !} d x d \mu(u) .
$$

It can be checked by elementary integrations by parts and induction on $k$, $r_{1}, \ldots, r_{k}$ that

$$
\int_{x \in \Delta_{k-1}} \prod_{1 \leq s \leq k} x_{s}^{r_{s}-1} d x_{1} \ldots d x_{k-1}=\frac{1}{(|r|-1) !} \prod_{1 \leq s \leq k}\left(r_{s}-1\right) ! .
$$

This implies that $(|r|-1) ! \prod_{1 \leq s \leq k} \frac{x_{s}^{r_{s}-1}}{\left(r_{s}-1\right) !} d x$ is a probability measure on $\Delta_{k-1}$ and that

$$
\lim _{p \rightarrow+\infty} I_{p}=\frac{1}{\prod_{s} a_{s}^{r_{s}}} \int_{u \in \prod S^{2 r_{s}-1}} f(u) d \mu(u) .
$$


Even without an explicit check, the evaluation (1.14) also follows from the fact that we must have equality for $f(z) \equiv 1$ in the latter equality, if we take into account the volume formula (1.11).

\section{Probabilistic estimate of the Curvature of $k$-Jet Bundles}

Let $(X, V)$ be a compact complex directed non singular variety. To avoid any technical difficulty at this point, we first assume that $V$ is a holomorphic vector subbundle of $T_{X}$, equipped with a smooth hermitian metric $h$.

According to the notation already specified in the introduction, we denote by $J^{k} V$ the bundle of $k$-jets of holomorphic curves $f:(\mathbb{C}, 0) \rightarrow X$ tangent to $V$ at each point. Let us set $n=\operatorname{dim}_{\mathbb{C}} X$ and $r=\operatorname{rank}_{\mathbb{C}} V$. Then $J^{k} V \rightarrow X$ is an algebraic fiber bundle with typical fiber $\mathbb{C}^{r k}$ (see below). It has a canonical $\mathbb{C}^{*}$-action defined by $\lambda \cdot f:(\mathbb{C}, 0) \rightarrow X,(\lambda \cdot f)(t)=f(\lambda t)$. Fix a point $x_{0}$ in $X$ and a local holomorphic coordinate system $\left(z_{1}, \ldots, z_{n}\right)$ centered at $x_{0}$ such that $V_{x_{0}}$ is the vector subspace $\left\langle\partial / \partial z_{1}, \ldots, \partial / \partial z_{r}\right\rangle$ at $x_{0}$. Then, in a neighborhood $U$ of $x_{0}, V$ admits a holomorphic frame of the form

$$
\frac{\partial}{\partial z_{\beta}}+\sum_{r+1 \leq \alpha \leq n} a_{\alpha \beta}(z) \frac{\partial}{\partial z_{\alpha}}, \quad 1 \leq \beta \leq r, a_{\alpha \beta}(0)=0 .
$$

Let $f(t)=\left(f_{1}(t), \ldots, f_{n}(t)\right)$ be a $k$-jet of curve tangent to $V$ starting from a point $f(0)=x \in U$. Such a curve is entirely determined by its initial point and by the projection $\widetilde{f}(t):=\left(f_{1}(t), \ldots, f_{r}(t)\right)$ to the first $r$-components, since the condition $f^{\prime}(t) \in V_{f(t)}$ implies that the other components must satisfy the ordinary differential equation

$$
f_{\alpha}^{\prime}(t)=\sum_{1 \leq \beta \leq r} a_{\alpha \beta}(f(t)) f_{\beta}^{\prime}(t) .
$$

This implies that the $k$-jet of $f$ is entirely determined by the initial point $x$ and the Taylor expansion

$$
\widetilde{f}(t)-\widetilde{x}=\xi_{1} t+\xi_{2} t^{2}+\ldots+\xi_{k} t^{k}+O\left(t^{k+1}\right)
$$

where $\xi_{s}=\left(\xi_{s \alpha}\right)_{1 \leq \alpha \leq r} \in \mathbb{C}^{r}$. The $\mathbb{C}^{*}$ action $(\lambda, f) \mapsto \lambda \cdot f$ is then expressed in coordinates by the weighted action

$$
\lambda \cdot\left(\xi_{1}, \xi_{2}, \ldots, \xi_{k}\right)=\left(\lambda \xi_{1}, \lambda^{2} \xi_{2}, \ldots, \lambda^{k} \xi_{k}\right)
$$


Holomorphic Morse Inequalities and The Green-Griffiths-Lang... 1181

associated with the weight $a=\left(1^{[r]}, 2^{[r]}, \ldots, k^{[r]}\right)$. The quotient projectived $k$-jet bundle

$$
X_{k}^{\mathrm{GG}}:=\left(J^{k} V \backslash\{0\}\right) / \mathbb{C}^{*}
$$

considered by Green and Griffiths [GG79] is therefore in a natural way a $P\left(1^{[r]}, 2^{[r]}, \ldots, k^{[r]}\right)$ weighted projective bundle over $X$. As such, it possesses a canonical sheaf $\mathcal{O}_{X_{k}^{\mathrm{GG}}}(1)$ such that $\mathcal{O}_{X_{k}^{\mathrm{GG}}}(m)$ is invertible when $m$ is a multiple of $\operatorname{lcm}(1,2, \ldots, k)$. Under the natural projection $\pi_{k}: X_{k}^{\mathrm{GG}} \rightarrow X$, the direct image $\left(\pi_{k}\right)_{*} \mathcal{O}_{X_{k}^{\mathrm{GG}}}(m)$ coincides with the sheaf of sections of the bundle $E_{k, m}^{\mathrm{GG}} V^{*}$ of jet differentials of order $k$ and degree $m$, namely polynomials

$$
P\left(z ; \xi_{1}, \ldots, \xi_{k}\right)=\sum_{\alpha_{\ell} \in \mathbb{N}^{r}, 1 \leq \ell \leq k} a_{\alpha_{1} \ldots \alpha_{k}}(z) \xi_{1}^{\alpha_{1}} \ldots \xi_{k}^{\alpha_{k}}
$$

of weighted degree $\left|\alpha_{1}\right|+2\left|\alpha_{2}\right|+\ldots+k\left|\alpha_{k}\right|=m$ on $J^{k} V$ with holomorphic coefficients. The jet differentials operate on germs of curves as differential operators

$$
P(f)(t)=\sum a_{\alpha_{1} \ldots \alpha_{k}}(f(t)) f^{\prime}(t)^{\alpha_{1}} \ldots f^{(k)}(t)^{\alpha_{k}}
$$

In the sequel, we do not make any further use of coordinate frames as (2.1), because they need not be related in any way to the hermitian metric $h$ of $V$. Instead, we choose a local holomorphic coordinate frame $\left(e_{\alpha}(z)\right)_{1 \leq \alpha \leq r}$ of $V$ on a neighborhood $U$ of $x_{0}$, such that

$$
\left\langle e_{\alpha}(z), e_{\beta}(z)\right\rangle=\delta_{\alpha \beta}+\sum_{1 \leq i, j \leq n, 1 \leq \alpha, \beta \leq r} c_{i j \alpha \beta} z_{i} \bar{z}_{j}+O\left(|z|^{3}\right)
$$

for suitable complex coefficients $\left(c_{i j \alpha \beta}\right)$. It is a standard fact that such a normalized coordinate system always exists, and that the Chern curvature tensor $\frac{i}{2 \pi} D_{V, h}^{2}$ of $(V, h)$ at $x_{0}$ is then given by

$$
\Theta_{V, h}\left(x_{0}\right)=-\frac{i}{2 \pi} \sum_{i, j, \alpha, \beta} c_{i j \alpha \beta} d z_{i} \wedge d \bar{z}_{j} \otimes e_{\alpha}^{*} \otimes e_{\beta} .
$$

Also, instead of defining the vectors $\xi_{s} \in \mathbb{C}^{r}$ as in (2.2), we consider a local holomorphic connection $\nabla$ on $V_{\mid U}$ (e.g. the one which turns $\left(e_{\alpha}\right)$ into a parallel frame), and take $\xi_{k}=\nabla^{k} f(0) \in V_{x}$ defined inductively by $\nabla^{1} f=f^{\prime}$ and $\nabla^{s} f=$ $\nabla_{f^{\prime}}\left(\nabla^{s-1} f\right)$. This is just another way of parametrizing the fibers of $J^{k} V$ over $U$ by the vector bundle $V_{\mid U}^{k}$. Notice that this is highly dependent on $\nabla$ (the bundle $J^{k} V$ actually does not carry a vector bundle or even affine bundle structure); however, the expression of the weighted action (2.3) is unchanged in this new setting. Now, we fix a finite open covering $\left(U_{\alpha}\right)_{\alpha \in I}$ of $X$ by open coordinate 
charts such that $V_{\mid U_{\alpha}}$ is trivial, along with holomorphic connections $\nabla_{\alpha}$ on $V_{\mid U_{\alpha}}$. Let $\theta_{\alpha}$ be a partition of unity of $X$ subordinate to the covering $\left(U_{\alpha}\right)$. Let us fix $p>0$ and small parameters $1=\varepsilon_{1} \gg \varepsilon_{2} \gg \ldots \gg \varepsilon_{k}>0$. Then we define a global weighted exhaustion on $J^{k} V$ by putting for any $k$-jet $f \in J_{x}^{k} V$

$$
\Psi_{h, p, \varepsilon}(f):=\left(\sum_{\alpha \in I} \theta_{\alpha}(x) \sum_{1 \leq s \leq k} \varepsilon_{s}^{2 p}\left\|\nabla_{\alpha}^{s} f(0)\right\|_{h(x)}^{2 p / s}\right)^{1 / p}
$$

where \|\|$_{h(x)}$ is the hermitian metric $h$ of $V$ evaluated on the fiber $V_{x}, x=f(0)$. The function $\Psi_{h, p, \varepsilon}$ satisfies the fundamental homogeneity property

$$
\Psi_{h, p, \varepsilon}(\lambda \cdot f)=\Psi_{h, p, \varepsilon}(f)|\lambda|^{2}
$$

with respect to the $\mathbb{C}^{*}$ action on $J^{k} V$, in other words, it induces a hermitian metric on the dual $L^{*}$ of the tautological $\mathbb{Q}$-line bundle $L_{k}=\mathcal{O}_{X_{k}^{\mathrm{GG}}}(1)$ over $X_{k}^{\mathrm{GG}}$. The curvature of $L_{k}$ is given by

$$
\pi_{k}^{*} \Theta_{L_{k}, \Psi_{h, p, \varepsilon}^{*}}=d d^{c} \log \Psi_{h, p, \varepsilon}
$$

where $\pi_{k}: J^{k} V \backslash\{0\} \rightarrow X_{k}^{\mathrm{GG}}$ is the canonical projection. Our next goal is to compute precisely the curvature and to apply holomorphic Morse inequalities to $L \rightarrow X_{k}^{\mathrm{GG}}$ with the above metric. It might look a priori like an untractable problem, since the definition of $\Psi_{h, p, \varepsilon}$ is a rather unnatural one. However, the "miracle" is that the asymptotic behavior of $\Psi_{h, p, \varepsilon}$ as $\varepsilon_{s} / \varepsilon_{s-1} \rightarrow 0$ is in some sense uniquely defined and very natural. It will lead to a computable asymptotic formula, which is moreover simple enough to produce useful results.

(2.12) Lemma. On each coordinate chart $U$ equipped with a holomorphic connection $\nabla$ of $V_{\mid U}$, let us define the components of a $k$-jet $f \in J^{k} V$ by $\xi_{s}=\nabla^{s} f(0)$, and consider the rescaling transformation

$$
\rho_{\varepsilon}\left(\xi_{1}, \xi_{2}, \ldots, \xi_{k}\right)=\left(\varepsilon_{1}^{1} \xi_{1}, \varepsilon_{2}^{2} \xi_{2}, \ldots, \varepsilon_{k}^{k} \xi_{k}\right) \quad \text { on } J_{x}^{k} V, x \in U_{\alpha}
$$

(it commutes with the $\mathbb{C}^{*}$-action but is otherwise unrelated and not canonically defined over $X$ as it depends on the choice of $\nabla)$. Then, if $p$ is a multiple of $\operatorname{lcm}(1,2, \ldots, k)$ and $\varepsilon_{s} / \varepsilon_{s-1} \rightarrow 0$ for all $s=2, \ldots, k$, the rescaled function $\Psi_{h, p, \varepsilon} \circ \rho_{\varepsilon}^{-1}\left(\xi_{1}, \ldots, \xi_{k}\right)$ converges towards

$$
\left(\sum_{1 \leq s \leq k}\left\|\xi_{s}\right\|_{h}^{2 p / s}\right)^{1 / p}
$$

on every compact subset of $J^{k} V_{\mid U} \backslash\{0\}$, uniformly in $C^{\infty}$ topology. 
Proof. Let $U \subset X$ be an open set on which $V_{\mid U}$ is trivial and equipped with some holomorphic connection $\nabla$. Let us pick another holomorphic connection $\widetilde{\nabla}=\nabla+\Gamma$ where $\Gamma \in H^{0}\left(U, \Omega_{X}^{1} \otimes \operatorname{Hom}(V, V)\right.$. Then $\widetilde{\nabla}^{2} f=\nabla^{2} f+\Gamma(f)\left(f^{\prime}\right) \cdot f^{\prime}$, and inductively we get

$$
\widetilde{\nabla}^{s} f=\nabla^{s} f+P_{s}\left(f ; \nabla^{1} f, \ldots, \nabla^{s-1} f\right)
$$

where $P\left(x ; \xi_{1}, \ldots, \xi_{s-1}\right)$ is a polynomial with holomorphic coefficients in $x \in U$ which is of weighted homogeneous degree $s$ in $\left(\xi_{1}, \ldots, \xi_{s-1}\right)$. In other words, the corresponding change in the parametrization of $J^{k} V_{\mid U}$ is given by a $\mathbb{C}^{*}$ homogeneous transformation

$$
\widetilde{\xi}_{s}=\xi_{s}+P_{s}\left(x ; \xi_{1}, \ldots, \xi_{s-1}\right) .
$$

Let us introduce the corresponding rescaled components

$$
\left(\xi_{1, \varepsilon}, \ldots, \xi_{k, \varepsilon}\right)=\left(\varepsilon_{1}^{1} \xi_{1}, \ldots, \varepsilon_{k}^{k} \xi_{k}\right), \quad\left(\widetilde{\xi}_{1, \varepsilon}, \ldots, \widetilde{\xi}_{k, \varepsilon}\right)=\left(\varepsilon_{1}^{1} \widetilde{\xi}_{1}, \ldots, \varepsilon_{k}^{k} \widetilde{\xi}_{k}\right) .
$$

Then

$$
\begin{aligned}
\widetilde{\xi}_{s, \varepsilon} & =\xi_{s, \varepsilon}+\varepsilon_{s}^{s} P_{s}\left(x ; \varepsilon_{1}^{-1} \xi_{1, \varepsilon}, \ldots, \varepsilon_{s-1}^{-(s-1)} \xi_{s-1, \varepsilon}\right) \\
& =\xi_{s, \varepsilon}+O\left(\varepsilon_{s} / \varepsilon_{s-1}\right)^{s} O\left(\left\|\xi_{1, \varepsilon}\right\|+\ldots+\left\|\xi_{s-1, \varepsilon}\right\|^{1 /(s-1)}\right)^{s}
\end{aligned}
$$

and the error terms are thus polynomials of fixed degree with arbitrarily small coefficients as $\varepsilon_{s} / \varepsilon_{s-1} \rightarrow 0$. Now, the definition of $\Psi_{h, p, \varepsilon}$ consists of glueing the sums

$$
\sum_{1 \leq s \leq k} \varepsilon_{s}^{2 p}\left\|\xi_{k}\right\|_{h}^{2 p / s}=\sum_{1 \leq s \leq k}\left\|\xi_{k, \varepsilon}\right\|_{h}^{2 p / s}
$$

corresponding to $\xi_{k}=\nabla_{\alpha}^{s} f(0)$ by means of the partition of unity $\sum \theta_{\alpha}(x)=1$. We see that by using the rescaled variables $\xi_{s, \varepsilon}$ the changes occurring when replacing a connection $\nabla_{\alpha}$ by an alternative one $\nabla_{\beta}$ are arbitrary small in $C^{\infty}$ topology, with error terms uniformly controlled in terms of the ratios $\varepsilon_{s} / \varepsilon_{s-1}$ on all compact subsets of $V^{k} \backslash\{0\}$. This shows that in $C^{\infty}$ topology, $\Psi_{h, p, \varepsilon} \circ \rho_{\varepsilon}^{-1}\left(\xi_{1}, \ldots, \xi_{k}\right)$ converges uniformly towards $\left(\sum_{1 \leq s \leq k}\left\|\xi_{k}\right\|_{h}^{2 p / s}\right)^{1 / p}$, whatever is the trivializing open set $U$ and the holomorphic connection $\nabla$ used to evaluate the components and perform the rescaling.

Now, we fix a point $x_{0} \in X$ and a local holomorphic frame $\left(e_{\alpha}(z)\right)_{1 \leq \alpha \leq r}$ satisfying (2.7) on a neighborhood $U$ of $x_{0}$. We introduce the rescaled components 
$\xi_{s}=\varepsilon_{s}^{s} \nabla^{s} f(0)$ on $J^{k} V_{\mid U}$ and compute the curvature of

$$
\Psi_{h, p, \varepsilon} \circ \rho_{\varepsilon}^{-1}\left(z ; \xi_{1}, \ldots, \xi_{k}\right) \simeq\left(\sum_{1 \leq s \leq k}\left\|\xi_{s}\right\|_{h}^{2 p / s}\right)^{1 / p}
$$

(by Lemma 2.12, the errors can be taken arbitrary small in $C^{\infty}$ topology). We write $\xi_{s}=\sum_{1 \leq \alpha \leq r} \xi_{s \alpha} e_{\alpha}$. By (2.7) we have

$$
\left\|\xi_{s}\right\|_{h}^{2}=\sum_{\alpha}\left|\xi_{s \alpha}\right|^{2}+\sum_{i, j, \alpha, \beta} c_{i j \alpha \beta} z_{i} \bar{z}_{j} \xi_{s \alpha} \bar{\xi}_{s \beta}+O\left(|z|^{3}|\xi|^{2}\right) .
$$

The question is to evaluate the curvature of the weighted metric defined by

$$
\begin{aligned}
\Psi\left(z ; \xi_{1}, \ldots, \xi_{k}\right) & =\left(\sum_{1 \leq s \leq k}\left\|\xi_{s}\right\|_{h}^{2 p / s}\right)^{1 / p} \\
& =\left(\sum_{1 \leq s \leq k}\left(\sum_{\alpha}\left|\xi_{s \alpha}\right|^{2}+\sum_{i, j, \alpha, \beta} c_{i j \alpha \beta} z_{i} \bar{z}_{j} \xi_{s \alpha} \bar{\xi}_{s \beta}\right)^{p / s}\right)^{1 / p}+O\left(|z|^{3}\right) .
\end{aligned}
$$

We set $\left|\xi_{s}\right|^{2}=\sum_{\alpha}\left|\xi_{s \alpha}\right|^{2}$. A straightforward calculation yields

$$
\begin{aligned}
& \log \Psi\left(z ; \xi_{1}, \ldots, \xi_{k}\right)= \\
& =\frac{1}{p} \log \sum_{1 \leq s \leq k}\left|\xi_{s}\right|^{2 p / s}+\sum_{1 \leq s \leq k} \frac{1}{s} \frac{\left|\xi_{s}\right|^{2 p / s}}{\sum_{t}\left|\xi_{t}\right|^{2 p / t}} \sum_{i, j, \alpha, \beta} c_{i j \alpha \beta} z_{i} \bar{z}_{j} \frac{\xi_{s \alpha} \bar{\xi}_{s \beta}}{\left|\xi_{s}\right|^{2}}+O\left(|z|^{3}\right) .
\end{aligned}
$$

By (2.11), the curvature form of $L_{k}=\mathcal{O}_{X_{k}^{G G}}(1)$ is given at the central point $x_{0}$ by the following formula.

(2.13) Proposition. With the above choice of coordinates and with respect to the rescaled components $\xi_{s}=\varepsilon_{s}^{s} \nabla^{s} f(0)$ at $x_{0} \in X$, we have the approximate expression

$$
\Theta_{L_{k}, \Psi_{h, p, \varepsilon}^{*}}\left(x_{0},[\xi]\right) \simeq \omega_{a, r, p}(\xi)+\frac{i}{2 \pi} \sum_{1 \leq s \leq k} \frac{1}{s} \frac{\left|\xi_{s}\right|^{2 p / s}}{\sum_{t}\left|\xi_{t}\right|^{2 p / t}} \sum_{i, j, \alpha, \beta} c_{i j \alpha \beta} \frac{\xi_{s \alpha} \bar{\xi}_{s \beta}}{\left|\xi_{s}\right|^{2}} d z_{i} \wedge d \bar{z}_{j}
$$

where the error terms are $O\left(\max _{2 \leq s \leq k}\left(\varepsilon_{s} / \varepsilon_{s-1}\right)^{s}\right)$ uniformly on the compact variety $X_{k}^{\mathrm{GG}}$. Here $\omega_{a, r, p}$ is the (degenerate) Kähler metric associated with the weight $a=\left(1^{[r]}, 2^{[r]}, \ldots, k^{[r]}\right)$ of the canonical $\mathbb{C}^{*}$ action on $J^{k} V$.

Thanks to the uniform approximation, we can (and will) neglect the error terms in the calculations below. Since $\omega_{a, r, p}$ is positive definite on the fibers of 
Holomorphic Morse Inequalities and The Green-Griffiths-Lang... 1185

$X_{k}^{\mathrm{GG}} \rightarrow X$ (at least outside of the axes $\xi_{s}=0$ ), the index of the $(1,1)$ curvature form $\Theta_{L_{k}, \Psi_{h, p, \varepsilon}^{*}}(z,[\xi])$ is equal to the index of the $(1,1)$-form

$$
\gamma_{k}(z, \xi):=\frac{i}{2 \pi} \sum_{1 \leq s \leq k} \frac{1}{s} \frac{\left|\xi_{s}\right|^{2 p / s}}{\sum_{t}\left|\xi_{t}\right|^{2 p / t}} \sum_{i, j, \alpha, \beta} c_{i j \alpha \beta}(z) \frac{\xi_{s \alpha} \bar{\xi}_{s \beta}}{\left|\xi_{s}\right|^{2}} d z_{i} \wedge d \bar{z}_{j}
$$

depending only on the differentials $\left(d z_{j}\right)_{1 \leq j \leq n}$ on $X$. The $q$-index integral of $\left(L_{k}, \Psi_{h, p, \varepsilon}^{*}\right)$ on $X_{k}^{\mathrm{GG}}$ is therefore equal to

$$
\begin{aligned}
& \int_{X_{k}^{\mathrm{GG}}\left(L_{k}, q\right)} \Theta_{L_{k}, \Psi_{h, p, \varepsilon}^{*}}^{n+k r-1}= \\
& =\frac{(n+k r-1) !}{n !(k r-1) !} \int_{z \in X} \int_{\xi \in P\left(1^{[r]}, \ldots, k[r]\right)} \omega_{a, r, p}^{k r-1}(\xi) \mathbb{1}_{\gamma_{k}, q}(z, \xi) \gamma_{k}(z, \xi)^{n}
\end{aligned}
$$

where $\mathbb{1}_{\gamma_{k}, q}(z, \xi)$ is the characteristic function of the open set of points where $\gamma_{k}(z, \xi)$ has signature $(n-q, q)$ in terms of the $d z_{j}$ 's. Notice that since $\gamma_{k}(z, \xi)^{n}$ is a determinant, the product $\mathbb{1}_{\gamma_{k}, q}(z, \xi) \gamma_{k}(z, \xi)^{n}$ gives rise to a continuous function on $X_{k}^{\mathrm{GG}}$. Formula 1.13 with $r_{1}=\ldots=r_{k}=r$ and $a_{s}=s$ yields the slightly more explicit integral

$$
\begin{aligned}
\int_{X_{k}^{\mathrm{GG}}\left(L_{k}, q\right)} & \Theta_{L_{k}, \Psi_{h, p, \varepsilon}^{*}}^{n+k r-1}=\frac{(n+k r-1) !}{n !(k !)^{r}} \times \\
& \int_{z \in X} \int_{(x, u) \in \Delta_{k-1} \times\left(S^{2 r-1}\right)^{k}} \mathbb{1}_{g_{k}, q}(z, x, u) g_{k}(z, x, u)^{n} \frac{\left(x_{1} \ldots x_{k}\right)^{r-1}}{(r-1) !^{k}} d x d \mu(u),
\end{aligned}
$$

where $g_{k}(z, x, u)=\gamma_{k}\left(z, x_{1}^{1 / 2 p} u_{1}, \ldots, x_{k}^{k / 2 p} u_{k}\right)$ is given by

$$
g_{k}(z, x, u)=\frac{i}{2 \pi} \sum_{1 \leq s \leq k} \frac{1}{s} x_{s} \sum_{i, j, \alpha, \beta} c_{i j \alpha \beta}(z) u_{s \alpha} \bar{u}_{s \beta} d z_{i} \wedge d \bar{z}_{j}
$$

and $\mathbb{1}_{g_{k}, q}(z, x, u)$ is the characteristic function of its $q$-index set. Here

$$
d \nu_{k, r}(x)=(k r-1) ! \frac{\left(x_{1} \ldots x_{k}\right)^{r-1}}{(r-1) !^{k}} d x
$$

is a probability measure on $\Delta_{k-1}$, and we can rewrite

$$
\begin{aligned}
& \int_{X_{k}^{\mathrm{GG}}\left(L_{k}, q\right)} \Theta_{L_{k}, \Psi_{h, p, \varepsilon}^{*}}^{n+k r-1}=\frac{(n+k r-1) !}{n !(k !)^{r}(k r-1) !} \times \\
& \quad \int_{z \in X} \int_{(x, u) \in \Delta_{k-1} \times\left(S^{2 r-1}\right)^{k}} \mathbb{1}_{g_{k}, q}(z, x, u) g_{k}(z, x, u)^{n} d \nu_{k, r}(x) d \mu(u) .
\end{aligned}
$$

Now, formula (2.15) shows that $g_{k}(z, x, u)$ is a "Monte Carlo" evaluation of the curvature tensor, obtained by averaging the curvature at random points 
$u_{s} \in S^{2 r-1}$ with certain positive weights $x_{s} / s$; we should then think of the $k$-jet $f$ as some sort of random parameter such that the derivatives $\nabla^{k} f(0)$ are uniformly distributed in all directions. Let us compute the expected value of $(x, u) \mapsto g_{k}(z, x, u)$ with respect to the probability measure $d \nu_{k, r}(x) d \mu(u)$. Since $\int_{S^{2 r-1}} u_{s \alpha} \bar{u}_{s \beta} d \mu\left(u_{s}\right)=\frac{1}{r} \delta_{\alpha \beta}$ and $\int_{\Delta_{k-1}} x_{s} d \nu_{k, r}(x)=\frac{1}{k}$, we find

$$
\mathbf{E}\left(g_{k}(z, \bullet, \bullet)\right)=\frac{1}{k r} \sum_{1 \leq s \leq k} \frac{1}{s} \cdot \frac{i}{2 \pi} \sum_{i, j, \alpha} c_{i j \alpha \alpha}(z) d z_{i} \wedge d \bar{z}_{j} .
$$

In other words, we get the normalized trace of the curvature, i.e.

$$
\mathbf{E}\left(g_{k}(z, \bullet, \bullet)\right)=\frac{1}{k r}\left(1+\frac{1}{2}+\ldots+\frac{1}{k}\right) \Theta_{\operatorname{det}\left(V^{*}\right), \operatorname{det} h^{*}},
$$

where $\Theta_{\operatorname{det}\left(V^{*}\right), \operatorname{det} h^{*}}$ is the $(1,1)$-curvature form of $\operatorname{det}\left(V^{*}\right)$ with the metric induced by $h$. It is natural to guess that $g_{k}(z, x, u)$ behaves asymptotically as its expected value $\mathbf{E}\left(g_{k}(z, \bullet, \bullet)\right)$ when $k$ tends to infinity. If we replace brutally $g_{k}$ by its expected value in (2.17), we get the integral

$$
\frac{(n+k r-1) !}{n !(k !)^{r}(k r-1) !} \frac{1}{(k r)^{n}}\left(1+\frac{1}{2}+\ldots+\frac{1}{k}\right)^{n} \int_{X} \mathbb{1}_{\eta, q} \eta^{n},
$$

where $\eta:=\Theta_{\operatorname{det}\left(V^{*}\right), \operatorname{det} h^{*}}$ and $\mathbb{1}_{\eta, q}$ is the characteristic function of its $q$-index set in $X$. The leading constant is equivalent to $(\log k)^{n} / n !(k !)^{r}$ modulo a multiplicative factor $1+O(1 / \log k)$. By working out a more precise analysis of the deviation, we will prove the following result.

(2.19) Probabilistic estimate. Fix smooth hermitian metrics $h$ on $V$ and $\omega=$ $\frac{i}{2 \pi} \sum \omega_{i j} d z_{i} \wedge d \bar{z}_{j}$ on $X$. Denote by $\Theta_{V, h}=-\frac{i}{2 \pi} \sum c_{i j \alpha \beta} d z_{i} \wedge d \bar{z}_{j} \otimes e_{\alpha}^{*} \otimes e_{\beta}$ the curvature tensor of $V$ with respect to an $h$-orthonormal frame $\left(e_{\alpha}\right)$, and put

$$
\eta(z)=\Theta_{\operatorname{det}\left(V^{*}\right), \operatorname{det} h^{*}}=\frac{i}{2 \pi} \sum_{1 \leq i, j \leq n} \eta_{i j} d z_{i} \wedge d \bar{z}_{j}, \quad \eta_{i j}=\sum_{1 \leq \alpha \leq r} c_{i j \alpha \alpha} .
$$

Finally consider the $k$-jet line bundle $L_{k}=\mathcal{O}_{X_{k}^{\mathrm{GG}}}(1) \rightarrow X_{k}^{\mathrm{GG}}$ equipped with the induced metric $\Psi_{h, p, \varepsilon}^{*}$ (as defined above, with $1=\varepsilon_{1} \gg \varepsilon_{2} \gg \ldots \gg \varepsilon_{k}>0$ ). When $k$ tends to infinity, the integral of the top power of the curvature of $L_{k}$ on its $q$-index set $X_{k}^{\mathrm{GG}}\left(L_{k}, q\right)$ is given by

$$
\int_{X_{k}^{\mathrm{GG}}\left(L_{k}, q\right)} \Theta_{L_{k}, \Psi_{h, p, \varepsilon}^{*}}^{n+k r-1}=\frac{(\log k)^{n}}{n !(k !)^{r}}\left(\int_{X} \mathbb{1}_{\eta, q} \eta^{n}+O\left((\log k)^{-1}\right)\right)
$$

for all $q=0,1, \ldots, n$, and the error term $O\left((\log k)^{-1}\right)$ can be bounded explicitly in terms of $\Theta_{V}, \eta$ and $\omega$. Moreover, the left hand side is identically zero for $q>n$. 
The final statement follows from the observation that the curvature of $L_{k}$ is positive along the fibers of $X_{k}^{\mathrm{GG}} \rightarrow X$, by the plurisubharmonicity of the weight (this is true even when the partition of unity terms are taken into account, since they depend only on the base); therefore the $q$-index sets are empty for $q>n$. We start with three elementary lemmas.

(2.20) Lemma. The integral

$$
I_{k, r, n}=\int_{\Delta_{k-1}}\left(\sum_{1 \leq s \leq k} \frac{x_{s}}{s}\right)^{n} d \nu_{k, r}(x)
$$

is given by the expansion

$$
\text { (a) } \quad I_{k, r, n}=\sum_{1 \leq s_{1}, s_{2}, \ldots, s_{n} \leq k} \frac{1}{s_{1} s_{2} \ldots s_{n}} \frac{(k r-1) !}{(r-1) !^{k}} \frac{\prod_{1 \leq i \leq k}\left(r-1+\beta_{i}\right) !}{(k r+n-1) !} .
$$

where $\beta_{i}=\beta_{i}(s)=\operatorname{card}\left\{j ; s_{j}=i\right\}, \sum \beta_{i}=n, 1 \leq i \leq k$. The quotient

$$
I_{k, r, n} / \frac{r^{n}}{k r(k r+1) \ldots(k r+n-1)}\left(1+\frac{1}{2}+\ldots+\frac{1}{k}\right)^{n}
$$

is bounded below by 1 and bounded above by

$$
\text { (b) } 1+\frac{1}{3} \sum_{m=2}^{n} \frac{2^{m} n !}{(n-m) !}\left(1+\frac{1}{2}+\ldots+\frac{1}{k}\right)^{-m}=1+O\left((\log k)^{-2}\right)
$$

As a consequence

$$
\text { (c) } \begin{aligned}
I_{k, r, n} & =\frac{1}{k^{n}}\left(\left(1+\frac{1}{2}+\ldots+\frac{1}{k}\right)^{n}+O\left((\log k)^{n-2}\right)\right) \\
& =\frac{(\log k+\gamma)^{n}+O\left((\log k)^{n-2}\right)}{k^{n}}
\end{aligned}
$$

where $\gamma$ is the Euler-Mascheroni constant.

Proof. Let us expand the $n$-th power $\left(\sum_{1 \leq s \leq k} \frac{x_{s}}{s}\right)^{n}$. This gives

$$
I_{k, r, n}=\sum_{1 \leq s_{1}, s_{2}, \ldots, s_{n} \leq k} \frac{1}{s_{1} s_{2} \ldots s_{n}} \int_{\Delta_{k-1}} x_{1}^{\beta_{1}} \ldots x_{k}^{\beta_{k}} d \nu_{k, r}(x)
$$

and by definition of the measure $\nu_{k, r}$ we have

$$
\int_{\Delta_{k-1}} x_{1}^{\beta_{1}} \ldots x_{k}^{\beta_{k}} d \nu_{k, r}(x)=\frac{(k r-1) !}{(r-1) !^{k}} \int_{\Delta_{k-1}} x_{1}^{r+\beta_{1}-1} \ldots x_{k}^{r+\beta_{k}-1} d x_{1} \ldots d x_{k} .
$$


By Formula (1.14), we find

$$
\begin{aligned}
\int_{\Delta_{k-1}} x_{1}^{\beta_{1}} \ldots x_{k}^{\beta_{k}} d \nu_{k, r}(x) & =\frac{(k r-1) !}{(r-1) !^{k}} \frac{\prod_{1 \leq i \leq k}\left(r+\beta_{i}-1\right) !}{(k r+n-1) !} \\
& =\frac{r^{n} \prod_{i, \beta_{i} \geq 1}\left(1+\frac{1}{r}\right)\left(1+\frac{2}{r}\right) \ldots\left(1+\frac{\beta_{i}-1}{r}\right)}{k r(k r+1) \ldots(k r+n-1)},
\end{aligned}
$$

and $(2.20 \mathrm{a})$ follows from the first equality. The final product is minimal when $r=1$, thus

$$
\begin{aligned}
\frac{r^{n}}{k r(k r+1) \ldots(k r+n-1)} & \leq \int_{\Delta_{k-1}} x_{1}^{\beta_{1}} \ldots x_{k}^{\beta_{k}} d \nu_{k, r}(x) \\
& \leq \frac{r^{n} \prod_{1 \leq i \leq k} \beta_{i} !}{k r(k r+1) \ldots(k r+n-1)} .
\end{aligned}
$$

Also, the integral is maximal when all $\beta_{i}$ vanish except one, in which case one gets

$$
\int_{\Delta_{k-1}} x_{j}^{n} d \nu_{k, r}(x)=\frac{r(r+1) \ldots(r+n-1)}{k r(k r+1) \ldots(k r+n-1)} .
$$

By (2.21), we find the lower and upper bounds

$$
\begin{aligned}
& I_{k, r, n} \geq \frac{r^{n}}{k r(k r+1) \ldots(k r+n-1)}\left(1+\frac{1}{2}+\ldots+\frac{1}{k}\right)^{n}, \\
& I_{k, r, n} \leq \frac{r^{n}}{k r(k r+1) \ldots(k r+n-1)} \sum_{1 \leq s_{1}, \ldots, s_{n} \leq k} \frac{\beta_{1} ! \ldots \beta_{k} !}{s_{1} \ldots s_{n}} .
\end{aligned}
$$

In order to make the upper bound more explicit, we reorganize the $n$-tuple $\left(s_{1}, \ldots, s_{n}\right)$ into those indices $t_{1}<\ldots<t_{\ell}$ which appear a certain number of times $\alpha_{i}=\beta_{t_{i}} \geq 2$, and those, say $t_{\ell+1}<\ldots<t_{\ell+m}$, which appear only once. We have of course $\sum \beta_{i}=n-m$, and each choice of the $t_{i}$ 's corresponds to $n ! / \alpha_{1} ! \ldots \alpha_{\ell}$ ! possibilities for the $n$-tuple $\left(s_{1}, \ldots, s_{n}\right)$. Therefore we get

$$
\sum_{1 \leq s_{1}, \ldots, s_{n} \leq k} \frac{\beta_{1} ! \ldots \beta_{k} !}{s_{1} \ldots s_{n}} \leq n ! \sum_{m=0}^{n} \sum_{\ell, \Sigma \alpha_{i}=n-m} \sum_{\left(t_{i}\right)} \frac{1}{t_{1}^{\alpha_{1}} \ldots t_{\ell}^{\alpha_{\ell}}} \frac{1}{t_{\ell+1} \ldots t_{\ell+m}} .
$$

A trivial comparison series vs. integral yields

$$
\sum_{s<t<+\infty} \frac{1}{t^{\alpha}} \leq \frac{1}{\alpha-1} \frac{1}{s^{\alpha-1}}
$$

and in this way, using successive integrations in $t_{\ell}, t_{\ell-1}, \ldots$, we get inductively

$$
\sum_{1 \leq t_{1}<\ldots<t_{\ell}<+\infty} \frac{1}{t_{1}^{\alpha_{1}} \ldots t_{\ell}^{\alpha_{\ell}}} \leq \frac{1}{\prod_{1 \leq i \leq \ell}\left(\alpha_{\ell-i+1}+\ldots+\alpha_{\ell}-i\right)} \leq \frac{1}{\ell !},
$$


since $\alpha_{i} \geq 2$ implies $\alpha_{\ell-i+1}+\ldots+\alpha_{\ell}-i \geq i$. On the other hand

$\sum_{1 \leq t_{\ell+1}<\ldots<t_{\ell+m} \leq k} \frac{1}{t_{\ell+1} \ldots t_{\ell+m}} \leq \frac{1}{m !} \sum_{1 \leq s_{1}, \ldots, s_{m} \leq k} \frac{1}{s_{1} \ldots s_{m}}=\frac{1}{m !}\left(1+\frac{1}{2}+\ldots+\frac{1}{k}\right)^{m}$.

Since partitions $\alpha_{1}+\ldots+\alpha_{\ell}=n-m$ satisfying the additional restriction $\alpha_{i} \geq 2$ correspond to $\alpha_{i}^{\prime}=\alpha_{i}-2$ satisfying $\sum \alpha_{i}^{\prime}=n-m-2 \ell$, their number is equal to

$$
\left(\begin{array}{c}
n-m-2 \ell+\ell-1 \\
\ell-1
\end{array}\right)=\left(\begin{array}{c}
n-m-\ell-1 \\
\ell-1
\end{array}\right) \leq 2^{n-m-\ell-1}
$$

and we infer from this

$\sum_{1 \leq s_{1}, \ldots, s_{n} \leq k} \frac{\beta_{1} ! \ldots \beta_{k} !}{s_{1} \ldots s_{n}} \leq \sum_{\substack{\ell \geq 1 \\ 2 \ell+m \leq n}} \frac{2^{n-m-\ell-1} n !}{\ell ! m !}\left(1+\frac{1}{2}+\ldots+\frac{1}{k}\right)^{m}+\left(1+\frac{1}{2}+\ldots+\frac{1}{k}\right)^{n}$

where the last term corresponds to the special case $\ell=0, m=n$. Therefore

$$
\begin{aligned}
\sum_{1 \leq s_{i} \leq k} \frac{\beta_{1} ! \ldots \beta_{k} !}{s_{1} \ldots s_{n}} & \leq \frac{e^{1 / 2}-1}{2} \sum_{m=0}^{n-2} \frac{2^{n-m} n !}{m !}\left(1+\frac{1}{2}+\ldots+\frac{1}{k}\right)^{m}+\left(1+\frac{1}{2}+\ldots+\frac{1}{k}\right)^{n} \\
& \leq \frac{1}{3} \sum_{m=2}^{n} \frac{2^{m} n !}{(n-m) !}\left(1+\frac{1}{2}+\ldots+\frac{1}{k}\right)^{n-m}+\left(1+\frac{1}{2}+\ldots+\frac{1}{k}\right)^{n} .
\end{aligned}
$$

This estimate combined with $(2.23,2.24)$ implies the upper bound $(2.20 \mathrm{~b})$ (the lower bound 1 being now obvious). The asymptotic estimate $(2.20 \mathrm{c})$ follows immediately.

(2.25) Lemma. If $A$ is a hermitian $n \times n$ matrix, set $\mathbb{1}_{A, q}$ to be equal to 1 if $A$ has signature $(n-q, q)$ and 0 otherwise. Then for all $n \times n$ hermitian matrices $A, B$ we have the estimate

$$
\left|\mathbb{1}_{A, q} \operatorname{det} A-\mathbb{1}_{B, q} \operatorname{det} B\right| \leq\|A-B\| \sum_{0 \leq i \leq n-1}\|A\|^{i}\|B\|^{n-1-i},
$$

where $\|A\|,\|B\|$ are the hermitian operator norms of the matrices.

Proof. We first check that the estimate holds true for $|\operatorname{det} A-\operatorname{det} B|$. Let $\lambda_{1} \leq$ $\ldots \leq \lambda_{n}$ be the eigenvalues of $A$ and $\lambda_{1}^{\prime} \leq \ldots \leq \lambda_{n}^{\prime}$ be the eigenvalues of $B$. We have $\left|\lambda_{i}\right| \leq\|A\|,\left|\lambda_{i}^{\prime}\right| \leq\|B\|$ and the minimax principle implies that $\left|\lambda_{i}-\lambda_{i}^{\prime}\right| \leq\|A-B\|$. We then get the desired estimate by writing

$$
\operatorname{det} A-\operatorname{det} B=\lambda_{1} \ldots \lambda_{n}-\lambda_{1}^{\prime} \ldots \lambda_{n}^{\prime}=\sum_{1 \leq i \leq n} \lambda_{1} \ldots \lambda_{i-1}\left(\lambda_{i}-\lambda_{i}^{\prime}\right) \lambda_{i+1}^{\prime} \ldots \lambda_{n}^{\prime} .
$$


This already implies (2.25) if $A$ or $B$ is degenerate. If $A$ and $B$ are non degenerate we only have to prove the result when one of them (say $A$ ) has signature $(n-q, q)$ and the other one (say $B$ ) has a different signature. If we put $M(t)=(1-t) A+t B$, the already established estimate for the determinant yields

$$
\left|\frac{d}{d t} \operatorname{det} M(t)\right| \leq n\|A-B\|\|M(t)\| \leq n\|A-B\|((1-t)\|A\|+t\|B\|)^{n-1} .
$$

However, since the signature of $M(t)$ is not the same for $t=0$ and $t=1$, there must exist $\left.t_{0} \in\right] 0,1\left[\operatorname{such}\right.$ that $\left(1-t_{0}\right) A+t_{0} B$ is degenerate. Our claim follows by integrating the differential estimate on the smallest such interval $\left[0, t_{0}\right]$, after observing that $M(0)=A$, $\operatorname{det} M\left(t_{0}\right)=0$, and that the integral of the right hand side on $[0,1]$ is the announced bound.

(2.26) Lemma. Let $Q_{A}$ be the hermitian quadratic form associated with the hermitian operator $A$ on $\mathbb{C}^{n}$. If $\mu$ is the rotation invariant probability measure on the unit sphere $S^{2 n-1}$ of $\mathbb{C}^{n}$ and $\lambda_{i}$ are the eigenvalues of $A$, we have

$$
\int_{|\zeta|=1}\left|Q_{A}(\zeta)\right|^{2} d \mu(\zeta)=\frac{1}{n(n+1)}\left(\sum \lambda_{i}^{2}+\left(\sum \lambda_{i}\right)^{2}\right) .
$$

The norm $\|A\|=\max \left|\lambda_{i}\right|$ satisfies the estimate

$$
\frac{1}{n^{2}}\|A\|^{2} \leq \int_{|\zeta|=1}\left|Q_{A}(\zeta)\right|^{2} d \mu(\zeta) \leq\|A\|^{2}
$$

Proof. The first identity as an easy calculation, and the inequalities follow by computing the eigenvalues of the quadratic form $\sum \lambda_{i}^{2}+\left(\sum \lambda_{i}\right)^{2}-c \lambda_{i_{0}}^{2}, c>0$. The lower bound is attained e.g. for $Q_{A}(\zeta)=\left|\zeta_{1}\right|^{2}-\frac{1}{n}\left(\left|\zeta_{2}\right|^{2}+\ldots+\left|\zeta_{n}\right|^{2}\right)$ when we take $i_{0}=1$ and $c=1+\frac{1}{n}$.

Proof of Proposition 2.19. Take a vector $\zeta \in T_{X, z}, \zeta=\sum \zeta_{i} \frac{\partial}{\partial z_{i}}$, with $\|\zeta\|_{\omega}=1$, and introduce the trace free sesquilinear quadratic form

$$
Q_{z, \zeta}(u)=\sum_{i, j, \alpha, \beta} \widetilde{c}_{i j \alpha \beta}(z) \zeta_{i} \bar{\zeta}_{j} u_{\alpha} \bar{u}_{\beta}, \quad \widetilde{c}_{i j \alpha \beta}=c_{i j \alpha \beta}-\frac{1}{r} \eta_{i j} \delta_{\alpha \beta}, \quad u \in \mathbb{C}^{r}
$$

where $\eta_{i j}=\sum_{1 \leq \alpha \leq r} c_{i j \alpha \alpha}$. We consider the corresponding trace free curvature tensor

$$
\widetilde{\Theta}_{V}=\frac{i}{2 \pi} \sum_{i, j, \alpha, \beta} \widetilde{c}_{i j \alpha \beta} d z_{i} \wedge d \bar{z}_{j} \otimes e_{\alpha}^{*} \otimes e_{\beta}
$$


As a general matter of notation, we adopt here the convention that the canonical correspondence between hermitian forms and (1,1)-forms is normalized as $\sum a_{i j} d z_{i} \otimes d \bar{z}_{j} \leftrightarrow \frac{i}{2 \pi} \sum a_{i j} d z_{i} \wedge d \bar{z}_{j}$, and we take the liberty of using the same symbols for both types of objects; we do so especially for $g_{k}(z, x, u)$ and $\eta(z)=$ $\frac{i}{2 \pi} \sum \eta_{i j}(z) d z_{i} \wedge d \bar{z}_{j}=\operatorname{Tr} \Theta_{V}(z)$. First observe that for all $k$-tuples of unit vectors $u=\left(u_{1}, \ldots, u_{k}\right) \in\left(S^{2 r-1}\right)^{k}, u_{s}=\left(u_{s \alpha}\right)_{1 \leq \alpha \leq r}$, we have

$$
\int_{\left(S^{2 r-1}\right)^{k}}\left|\sum_{1 \leq s \leq k} \frac{1}{s} x_{s} \sum_{i, j, \alpha, \beta} \widetilde{c}_{i j \alpha \beta}(z) \zeta_{i} \bar{\zeta}_{j} u_{s \alpha} \bar{u}_{s \beta}\right|^{2} d \mu(u)=\sum_{1 \leq s \leq k} \frac{x_{s}^{2}}{s^{2}} \mathbf{V}\left(Q_{z, \zeta}\right)
$$

where $\mathbf{V}\left(Q_{z, \zeta}\right)$ is the variance of $Q_{z, \zeta}$ on $S^{2 r-1}$. This is so because we have a sum over $s$ of independent random variables on $\left(S^{2 r-1}\right)^{k}$, all of which have zero mean value. (Lemma 2.26 shows that the variance $\mathbf{V}(Q)$ of a trace free hermitian quadratic form $Q(u)=\sum_{1 \leq \alpha \leq r} \lambda_{\alpha}\left|u_{\alpha}\right|^{2}$ on the unit sphere $S^{2 r-1}$ is equal to $\frac{1}{r(r+1)} \sum \lambda_{\alpha}^{2}$, but we only give the formula to fix the ideas). Formula (2.22) yields

$$
\int_{\Delta_{k-1}} x_{s}^{2} d \nu_{k, r}(x)=\frac{r+1}{k(k r+1)} .
$$

Therefore, according to notation (2.15), we obtain the partial variance formula

$$
\begin{aligned}
& \int_{\Delta_{k-1} \times\left(S^{2 r-1}\right)^{k}}\left|g_{k}(z, x, u)(\zeta)-\bar{g}_{k}(z, x)(\zeta)\right|^{2} d \nu_{k, r}(x) d \mu(u) \\
& \quad=\frac{(r+1)}{k(k r+1)}\left(\sum_{1 \leq s \leq k} \frac{1}{s^{2}}\right) \sigma_{h}\left(\widetilde{\Theta}_{V}(\zeta, \zeta)\right)^{2}
\end{aligned}
$$

in which

$$
\begin{aligned}
\bar{g}_{k}(z, x)(\zeta) & =\sum_{1 \leq s \leq k} \frac{1}{s} x_{s} \frac{1}{r} \sum_{i j \alpha} c_{i j \alpha \alpha} \zeta_{i} \bar{\zeta}_{j}=\left(\sum_{1 \leq s \leq k} \frac{1}{s} x_{s}\right) \frac{1}{r} \eta(z)(\zeta), \\
\sigma_{h}\left(\widetilde{\Theta}_{V}(\zeta, \zeta)\right)^{2} & =\mathbf{V}\left(u \mapsto\left\langle\widetilde{\Theta}_{V}(\zeta, \zeta) u, u\right\rangle_{h}\right)=\int_{u \in S^{2 r-1}}\left|\left\langle\widetilde{\Theta}_{V}(\zeta, \zeta) u, u\right\rangle_{h}\right|^{2} d \mu(u) .
\end{aligned}
$$

By integrating over $\zeta \in S^{2 n-1} \subset \mathbb{C}^{n}$ and applying the left hand inequality in Lemma 2.26 we infer

$$
\begin{aligned}
& \int_{\Delta_{k-1} \times\left(S^{2 r-1}\right)^{k}}\left\|g_{k}(z, x, u)-\bar{g}_{k}(z, x)\right\|_{\omega}^{2} d \nu_{k, r}(x) d \mu(u) \\
& \quad \leq \frac{n^{2}(r+1)}{k(k r+1)}\left(\sum_{1 \leq s \leq k} \frac{1}{s^{2}}\right) \sigma_{\omega, h}\left(\widetilde{\Theta}_{V}\right)^{2}
\end{aligned}
$$


where $\sigma_{\omega, h}\left(\widetilde{\Theta}_{V}\right)$ is the standard deviation of $\widetilde{\Theta}_{V}$ on $S^{2 n-1} \times S^{2 r-1}$ :

$$
\sigma_{\omega, h}\left(\widetilde{\Theta}_{V}\right)^{2}=\int_{|\zeta|_{\omega}=1,|u|_{h}=1}\left|\left\langle\widetilde{\Theta}_{V}(\zeta, \zeta) u, u\right\rangle_{h}\right|^{2} d \mu(\zeta) d \mu(u) .
$$

On the other hand, brutal estimates give the hermitian operator norm estimates

$$
\begin{aligned}
\left\|\bar{g}_{k}(z, x)\right\|_{\omega} & \leq\left(\sum_{1 \leq s \leq k} \frac{1}{s} x_{s}\right) \frac{1}{r}\|\eta(z)\|_{\omega}, \\
\left\|g_{k}(z, x, u)\right\|_{\omega} & \leq\left(\sum_{1 \leq s \leq k} \frac{1}{s} x_{s}\right)\left\|\Theta_{V}\right\|_{\omega, h}
\end{aligned}
$$

where

$$
\left\|\Theta_{V}\right\|_{\omega, h}=\sup _{|\zeta|_{\omega}=1,|u|_{h}=1}\left|\left\langle\Theta_{V}(\zeta, \zeta) u, u\right\rangle_{h}\right| .
$$

We use these estimates to evaluate the $q$-index integrals. The integral associated with $\bar{g}_{k}(z, x)$ is much easier to deal with than $g_{k}(z, x, u)$ since the characteristic function of the $q$-index set depends only on $z$. By Lemma 2.25 we find

$$
\begin{aligned}
& \left|\mathbb{1}_{g_{k}, q}(z, x, u) \operatorname{det} g_{k}(z, x, u)-\mathbb{1}_{\eta, q}(z) \operatorname{det} \bar{g}_{k}(z, x)\right| \\
& \quad \leq\left\|g_{k}(z, x, u)-\bar{g}_{k}(z, x)\right\|_{\omega} \sum_{0 \leq i \leq n-1}\left\|g_{k}(z, x, u)\right\|_{\omega}^{i}\left\|\bar{g}_{k}(z, x)\right\|_{\omega}^{n-1-i} .
\end{aligned}
$$

The Cauchy-Schwarz inequality combined with $(2.28-2.30)$ implies

$$
\begin{aligned}
& \int_{\Delta_{k-1} \times\left(S^{2 r-1}\right)^{k}}\left|\mathbb{1}_{g_{k}, q}(z, x, u) \operatorname{det} g_{k}(z, x, u)-\mathbb{1}_{\eta, q}(z) \operatorname{det} \bar{g}_{k}(z, x)\right| d \nu_{k, r}(x) d \mu(u) \\
& \leq\left(\int_{\Delta_{k-1} \times\left(S^{2 r-1}\right)^{k}}\left\|g_{k}(z, x, u)-\bar{g}_{k}(z, x)\right\|_{\omega}^{2} d \nu_{k, r}(x) d \mu(u)\right)^{1 / 2} \times \\
&\left(\int_{\Delta_{k-1} \times\left(S^{2 r-1}\right)^{k}}\left(\sum_{0 \leq i \leq n-1}\left\|g_{k}(z, x, u)\right\|_{\omega}^{i}\left\|\bar{g}_{k}(z, x)\right\|_{\omega}^{n-1-i}\right)^{2} d \nu_{k, r}(x) d \mu(u)\right)^{1 / 2} \\
& \leq \frac{n(1+1 / r)^{1 / 2}}{(k(k+1 / r))^{1 / 2}}\left(\sum_{1 \leq s \leq k} \frac{1}{s^{2}}\right)^{1 / 2} \sigma_{\omega, h}\left(\widetilde{\Theta}_{V}\right) \sum_{1 \leq i \leq n-1}\left\|\Theta_{V}\right\|_{\omega, h}^{i}\left(\frac{1}{r}\|\eta(z)\|_{\omega}\right)^{n-1-i} \\
& \times\left(\int_{\Delta_{k-1}}\left(\sum_{1 \leq s \leq k} \frac{x_{s}}{s}\right)^{2 n-2} d \nu_{k, r}(x)\right)^{1 / 2}=O\left(\frac{(\log k)^{n-1}}{k^{n}}\right)
\end{aligned}
$$

by Lemma 2.20 with $n$ replaced by $2 n-2$. This is the essential error estimate. As one can see, the growth of the error mainly depends on the final integral factor, 
since the initial multiplicative factor is uniformly bounded over $X$. In order to get the principal term, we compute

$$
\begin{aligned}
\int_{\Delta_{k-1}} \operatorname{det} \bar{g}_{k}(z, x) d \nu_{k, r}(x) & =\frac{1}{r^{n}} \operatorname{det} \eta(z) \int_{\Delta_{k-1}}\left(\sum_{1 \leq s \leq k} \frac{x_{s}}{s}\right)^{n} d \nu_{k, r}(x) \\
& \sim \frac{(\log k)^{n}}{r^{n} k^{n}} \operatorname{det} \eta(z) .
\end{aligned}
$$

From there we conclude that

$$
\begin{aligned}
& \int_{z \in X} \int_{(x, u) \in \Delta_{k-1} \times\left(S^{2 r-1}\right)^{k}} \mathbb{1}_{g_{k}, q}(z, x, u) g_{k}(z, x, u)^{n} d \nu_{k, r}(x) d \mu(u) \\
& =\frac{(\log k)^{n}}{r^{n} k^{n}} \int_{X} \mathbb{1}_{\eta, q} \eta^{n}+O\left(\frac{(\log k)^{n-1}}{k^{n}}\right)
\end{aligned}
$$

The probabilistic estimate 2.19 follows by (2.17).

(2.31) Remark. If we take care of the precise bounds obtained above, the proof gives in fact the explicit estimate

$$
\int_{X_{k}^{\mathrm{GG}}\left(L_{k}, q\right)} \Theta_{L_{k}, \Psi_{h, p, \varepsilon}^{n}}^{n+k r-1}=\frac{(n+k r-1) ! I_{k, r, n}}{n !(k !)^{r}(k r-1) !}\left(\int_{X} \mathbb{1}_{\eta, q} \eta^{n}+\varepsilon_{k, r, n} J\right)
$$

where

$$
J=n(1+1 / r)^{1 / 2}\left(\sum_{s=1}^{k} \frac{1}{s^{2}}\right)^{1 / 2} \int_{X} \sigma_{\omega, h}\left(\widetilde{\Theta}_{V}\right) \sum_{i=1}^{n-1} r^{i+1}\left\|\Theta_{V}\right\|_{\omega, h}^{i}\|\eta(z)\|_{\omega}^{n-1-i} \omega^{n}
$$

and

$$
\begin{aligned}
\left|\varepsilon_{k, r, n}\right| \leq & \left(\int_{\Delta_{k-1}}\left(\sum_{s=1}^{k} \frac{x_{s}}{s}\right)^{2 n-2} d \nu_{k, r}(x)\right)^{1 / 2} \\
& \leq \frac{\left(1+\frac{1}{3} \sum_{m=2}^{2 n-2} \frac{2^{m}(2 n-2) !}{(2 n-2-m) !}\left(1+\frac{1}{2}+\ldots+\frac{1}{k}\right)^{-m}\right)^{1 / 2}}{1+\frac{1}{2}+\ldots+\frac{1}{k}} \sim \frac{1}{\log k}
\end{aligned}
$$

by the lower and upper bounds of $I_{k, r, n}, I_{k, r, 2 n-2}$ obtained in Lemma 2.20. As $(2 n-2) ! /(2 n-2-m) ! \leq(2 n-2)^{m}$, one easily shows that

$$
\left|\varepsilon_{k, r, n}\right| \leq \frac{(31 / 15)^{1 / 2}}{\log k} \quad \text { for } k \geq e^{5 n-5} .
$$


Also, we see that the error terms vanish if $\widetilde{\Theta}_{V}$ is identically zero, but this is of course a rather unexpected circumstance. In general, since the form $\widetilde{\Theta}_{V}$ is trace free, Lemma 2.23 applied to the quadratic form $u \mapsto\left\langle\widetilde{\Theta}_{V}(\zeta, \zeta) u, u\right\rangle$ on $\mathbb{C}^{r}$ implies $\sigma_{\omega, h}\left(\widetilde{\Theta}_{V}\right) \leq(r+1)^{-1 / 2}\left\|\widetilde{\Theta}_{V}\right\|_{\omega, h}$. This yields the simpler bound

$$
J \leq n r^{1 / 2}\left(\sum_{s=1}^{k} \frac{1}{s^{2}}\right)^{1 / 2} \int_{X}\left\|\widetilde{\Theta}_{V}\right\|_{\omega, h} \sum_{i=1}^{n-1} r^{i}\left\|\Theta_{V}\right\|_{\omega, h}^{i}\|\eta(z)\|_{\omega}^{n-1-i} \omega^{n} .
$$

It will be useful to extend the above estimates to the case of sections of

$$
L_{k}=\mathcal{O}_{X_{k}^{G G}}(1) \otimes \pi_{k}^{*} \mathcal{O}\left(\frac{1}{k r}\left(1+\frac{1}{2}+\ldots+\frac{1}{k}\right) F\right)
$$

where $F \in \operatorname{Pic}_{\mathbb{Q}}(X)$ is an arbitrary $\mathbb{Q}$-line bundle on $X$ and $\pi_{k}: X_{k}^{\mathrm{GG}} \rightarrow X$ is the natural projection. We assume here that $F$ is also equipped with a smooth hermitian metric $h_{F}$. In formula (2.20), the renormalized metric $\eta_{k}(z, x, u)$ of $L_{k}$ takes the form

$$
\eta_{k}(z, x, u)=\frac{1}{\frac{1}{k r}\left(1+\frac{1}{2}+\ldots+\frac{1}{k}\right)} g_{k}(z, x, u)+\Theta_{F, h_{F}}(z),
$$

and by the same calculations its expected value is

$$
\eta(z):=\mathbf{E}\left(\eta_{k}(z, \bullet, \bullet)\right)=\Theta_{\operatorname{det} V^{*}, \operatorname{det} h^{*}}(z)+\Theta_{F, h_{F}}(z) .
$$

Then the variance estimate for $\eta_{k}-\eta$ is unchanged, and the $L^{p}$ bounds for $\eta_{k}$ are still valid, since our forms are just shifted by adding the constant smooth term $\Theta_{F, h_{F}}(z)$. The probabilistic estimate 2.18 is therefore still true in exactly the same form, provided we use $(2.34-2.36)$ instead of the previously defined $L_{k}, \eta_{k}$ and $\eta$. An application of holomorphic Morse inequalities gives the desired cohomology estimates for

$$
\begin{aligned}
h^{q} & \left(X, E_{k, m}^{\mathrm{GG}} V^{*} \otimes \mathcal{O}\left(\frac{m}{k r}\left(1+\frac{1}{2}+\ldots+\frac{1}{k}\right) F\right)\right) \\
\quad & =h^{q}\left(X_{k}^{\mathrm{GG}}, \mathcal{O}_{X_{k}^{\mathrm{GG}}}(m) \otimes \pi_{k}^{*} \mathcal{O}\left(\frac{m}{k r}\left(1+\frac{1}{2}+\ldots+\frac{1}{k}\right) F\right)\right),
\end{aligned}
$$

provided $m$ is sufficiently divisible to give a multiple of $F$ which is a $\mathbb{Z}$-line bundle.

(2.37) Theorem. Let $(X, V)$ be a directed manifold, $F \rightarrow X$ a $\mathbb{Q}$-line bundle, $(V, h)$ and $\left(F, h_{F}\right)$ smooth hermitian structure on $V$ and $F$ respectively. We 
define

$$
\begin{aligned}
L_{k} & =\mathcal{O}_{X_{k}^{\mathrm{GG}}}(1) \otimes \pi_{k}^{*} \mathcal{O}\left(\frac{1}{k r}\left(1+\frac{1}{2}+\ldots+\frac{1}{k}\right) F\right), \\
\eta & =\Theta_{\operatorname{det} V^{*}, \operatorname{det} h^{*}}+\Theta_{F, h_{F}} .
\end{aligned}
$$

Then for all $q \geq 0$ and all $m \gg k \gg 1$ such that $m$ is sufficiently divisible, we have
(a) $h^{q}\left(X_{k}^{\mathrm{GG}}, \mathcal{O}\left(L_{k}^{\otimes m}\right)\right) \leq \frac{m^{n+k r-1}}{(n+k r-1) !} \frac{(\log k)^{n}}{n !(k !)^{r}}\left(\int_{X(\eta, q)}(-1)^{q} \eta^{n}+O\left((\log k)^{-1}\right)\right)$,
(b) $h^{0}\left(X_{k}^{\mathrm{GG}}, \mathcal{O}\left(L_{k}^{\otimes m}\right)\right) \geq \frac{m^{n+k r-1}}{(n+k r-1) !} \frac{(\log k)^{n}}{n !(k !)^{r}}\left(\int_{X(\eta, \leq 1)} \eta^{n}-O\left((\log k)^{-1}\right)\right)$,
(c) $\chi\left(X_{k}^{\mathrm{GG}}, \mathcal{O}\left(L_{k}^{\otimes m}\right)\right)=\frac{m^{n+k r-1}}{(n+k r-1) !} \frac{(\log k)^{n}}{n !(k !)^{r}}\left(c_{1}\left(V^{*} \otimes F\right)^{n}+O\left((\log k)^{-1}\right)\right)$.

Green and Griffiths [GG79] already checked the Riemann-Roch calculation $(2.37 \mathrm{c})$ in the special case $V=T_{X}^{*}$ and $F=\mathcal{O}_{X}$. Their proof is much simpler since it relies only on Chern class calculations, but it cannot provide any information on the individual cohomology groups, except in very special cases where vanishing theorems can be applied; in fact in dimension 2, the Euler characteristic satisfies $\chi=h^{0}-h^{1}+h^{2} \leq h^{0}+h^{2}$, hence it is enough to get the vanishing of the top cohomology group $H^{2}$ to infer $h^{0} \geq \chi$; this works for surfaces by means of a well-known vanishing theorem of Bogomolov which implies in general

$$
\left.H^{n}\left(X, E_{k, m}^{\mathrm{GG}} T_{X}^{*} \otimes \mathcal{O}\left(\frac{m}{k r}\left(1+\frac{1}{2}+\ldots+\frac{1}{k}\right) F\right)\right)\right)=0
$$

as soon as $K_{X} \otimes F$ is big and $m \gg 1$.

In fact, thanks to Bonavero's singular holomorphic Morse inequalities [Bon93], everything works almost unchanged in the case where $V \subset T_{X}$ has singularities and $h$ is an admissible metric on $V$ (see (0.11)). We only have to find a blowup $\mu: \widetilde{X}_{k} \rightarrow X_{k}$ so that the resulting pull-backs $\mu^{*} L_{k}$ and $\mu^{*} V$ are locally free, and $\mu^{*} \operatorname{det} h^{*}, \mu^{*} \Psi_{h, p, \varepsilon}$ only have divisorial singularities. Then $\eta$ is a $(1,1)$ current with logarithmic poles, and we have to deal with smooth metrics on $\mu^{*} L_{k}^{\otimes m} \otimes \mathcal{O}\left(-m E_{k}\right)$ where $E_{k}$ is a certain effective divisor on $X_{k}$ (which, by our assumption (0.11), does not project onto $X)$. The cohomology groups involved are then the twisted cohomology groups

$$
H^{q}\left(X_{k}^{\mathrm{GG}}, \mathcal{O}\left(L_{k}^{\otimes m}\right) \otimes \mathcal{J}_{k, m}\right)
$$


where $\mathcal{J}_{k, m}=\mu_{*}\left(\mathcal{O}\left(-m E_{k}\right)\right)$ is the corresponding multiplier ideal sheaf, and the Morse integrals need only be evaluated in the complement of the poles, that is on $X(\eta, q) \backslash S$ where $S=\operatorname{Sing}(V) \cup \operatorname{Sing}(h)$. Since

$$
\left.\left(\pi_{k}\right)_{*}\left(\mathcal{O}\left(L_{k}^{\otimes m}\right) \otimes \mathcal{J}_{k, m}\right) \subset E_{k, m}^{\mathrm{GG}} V^{*} \otimes \mathcal{O}\left(\frac{m}{k r}\left(1+\frac{1}{2}+\ldots+\frac{1}{k}\right) F\right)\right)
$$

we still get a lower bound for the $H^{0}$ of the latter sheaf (or for the $H^{0}$ of the un-twisted line bundle $\mathcal{O}\left(L_{k}^{\otimes m}\right)$ on $\left.X_{k}^{\mathrm{GG}}\right)$. If we assume that $K_{V} \otimes F$ is big, these considerations also allow us to obtain a strong estimate in terms of the volume, by using an approximate Zariski decomposition on a suitable blow-up of $(X, V)$. The following corollary implies in particular Theorem 0.5.

(2.38) Corollary. If $F$ is an arbitrary $\mathbb{Q}$-line bundle over $X$, one has

$$
\begin{aligned}
& h^{0}\left(X_{k}^{\mathrm{GG}}, \mathcal{O}_{X_{k}^{\mathrm{GG}}}(m) \otimes \pi_{k}^{*} \mathcal{O}\left(\frac{m}{k r}\left(1+\frac{1}{2}+\ldots+\frac{1}{k}\right) F\right)\right) \\
& \quad \geq \frac{m^{n+k r-1}}{(n+k r-1) !} \frac{(\log k)^{n}}{n !(k !)^{r}}\left(\operatorname{Vol}\left(K_{V} \otimes F\right)-O\left((\log k)^{-1}\right)\right)-o\left(m^{n+k r-1}\right),
\end{aligned}
$$

when $m \gg k \gg 1$, in particular there are many sections of the $k$-jet differentials of degree $m$ twisted by the appropriate power of $F$ if $K_{V} \otimes F$ is big.

Proof. The volume is computed here as usual, i.e. after performing a suitable modification $\mu: \widetilde{X} \rightarrow X$ which converts $K_{V}$ into an invertible sheaf. There is of course nothing to prove if $K_{V} \otimes F$ is not big, so we can assume $\operatorname{Vol}\left(K_{V} \otimes F\right)>0$. Let us fix smooth hermitian metrics $h_{0}$ on $T_{X}$ and $h_{F}$ on $F$. They induce a metric $\mu^{*}\left(\operatorname{det} h_{0}^{-1} \otimes h_{F}\right)$ on $\mu^{*}\left(K_{V} \otimes F\right)$ which, by our definition of $K_{V}$, is a smooth metric. By the result of Fujita [Fuj94] on approximate Zariski decomposition, for every $\delta>0$, one can find a modification $\mu_{\delta}: \widetilde{X}_{\delta} \rightarrow X$ dominating $\mu$ such that

$$
\mu_{\delta}^{*}\left(K_{V} \otimes F\right)=\mathcal{O}_{\widetilde{X}_{\delta}}(A+E)
$$

where $A$ and $E$ are $\mathbb{Q}$-divisors, $A$ ample and $E$ effective, with

$$
\operatorname{Vol}(A)=A^{n} \geq \operatorname{Vol}\left(K_{V} \otimes F\right)-\delta .
$$

If we take a smooth metric $h_{A}$ with positive definite curvature form $\Theta_{A, h_{A}}$, then we get a singular hermitian metric $h_{A} h_{E}$ on $\mu_{\delta}^{*}\left(K_{V} \otimes F\right)$ with poles along $E$, i.e. the quotient $h_{A} h_{E} / \mu^{*}\left(\operatorname{det} h_{0}^{-1} \otimes h_{F}\right)$ is of the form $e^{-\varphi}$ where $\varphi$ is quasi-psh with $\log$ poles $\log \left|\sigma_{E}\right|^{2}\left(\bmod C^{\infty}\left(\widetilde{X}_{\delta}\right)\right)$ precisely given by the divisor $E$. We then only 
Holomorphic Morse Inequalities and The Green-Griffiths-Lang... 1197

need to take the singular metric $h$ on $T_{X}$ defined by

$$
h=h_{0} e^{\frac{1}{r}\left(\mu_{\delta}\right) * \varphi}
$$

(the choice of the factor $\frac{1}{r}$ is there to correct adequately the metric on $\operatorname{det} V$ ). By construction $h$ induces an admissible metric on $V$ and the resulting curvature current $\eta=\Theta_{K_{V}, \operatorname{det} h^{*}}+\Theta_{F, h_{F}}$ is such that

$$
\mu_{\delta}^{*} \eta=\Theta_{A, h_{A}}+[E], \quad[E]=\text { current of integration on } E .
$$

Then the 0-index Morse integral in the complement of the poles is given by

$$
\int_{X(\eta, 0) \backslash S} \eta^{n}=\int_{\widetilde{X}_{\delta}} \Theta_{A, h_{A}}^{n}=A^{n} \geq \operatorname{Vol}\left(K_{V} \otimes F\right)-\delta
$$

and (2.38) follows from the fact that $\delta$ can be taken arbitrary small.

(2.39) Example. In some simple cases, the above estimates can lead to very explicit results. Take for instance $X$ to be a smooth complete intersection of multidegree $\left(d_{1}, d_{2}, \ldots, d_{s}\right)$ in $\mathbb{P}_{\mathbb{C}}^{n+s}$ and consider the absolute case $V=T_{X}$. Then

$$
K_{X}=\mathcal{O}_{X}\left(d_{1}+\ldots+d_{s}-n-s-1\right) .
$$

Assume that $X$ is of general type, i.e. $\sum d_{j}>n+s+1$. Let us equip $V=T_{X}$ with the restriction of the Fubini-Study metric $h=\Theta_{\mathcal{O}(1)}$; a better choice might be the Kähler-Einstein metric but we want to keep the calculations as elementary as possible. The standard formula for the curvature tensor of a submanifold gives

$$
\Theta_{T_{X}, h}=\left(\Theta_{T_{\mathbb{P} n+s}, h}\right)_{\mid X}+\beta^{*} \wedge \beta
$$

where $\beta \in C^{\infty}\left(\Lambda^{1,0} T_{X}^{*} \otimes \operatorname{Hom}\left(T_{X}, \bigoplus \mathcal{O}\left(d_{j}\right)\right)\right)$ is the second fundamental form. In other words, by the well known formula for the curvature of projective space, we have

$$
\left\langle\Theta_{T_{X}, h}(\zeta, \zeta) u, u\right\rangle=|\zeta|^{2}|u|^{2}+|\langle\zeta, u\rangle|^{2}-|\beta(\zeta) \cdot u|^{2} .
$$

The curvature $\rho$ of $\left(K_{X}\right.$, det $\left.h^{*}\right)$ (i.e. the opposite of the Ricci form $\left.\operatorname{Tr} \Theta_{T_{X}, h}\right)$ is given by

$$
\rho=-\operatorname{Tr} \Theta_{T X, h}=\operatorname{Tr}\left(\beta \wedge \beta^{*}\right)-(n+1) h \geq-(n+1) h .
$$

We take here $F=\mathcal{O}_{X}(-a), a \in \mathbb{Q}_{+}$, and we want to determine conditions for the existence of sections

$$
H^{0}\left(X, E_{k, m}^{\mathrm{GG}} T_{X}^{*} \otimes \mathcal{O}\left(-a \frac{m}{k r}\left(1+\frac{1}{2}+\ldots+\frac{1}{k}\right)\right)\right), \quad m \gg 1 .
$$


We have to choose $K_{X} \otimes \mathcal{O}_{X}(-a)$ ample, i.e. $\sum d_{j}>n+s+a+1$, and then (by an appropriate choice of the metric of $F=\mathcal{O}_{X}(-a)$ ), the form $\eta=\Theta_{K_{X} \otimes \mathcal{O}_{X}(-a)}$ can be taken to be any positive form cohomologous to $\left(\sum d_{j}-(n+s+a+1)\right) h$. We use remark 2.31 and estimate the error terms by considering the Kähler metric

$$
\omega=\rho+(n+s+2) h \equiv\left(\sum d_{j}+1\right) h .
$$

Inequality (2.40) shows that $\omega \geq 2 h$ and also that $\omega \geq \operatorname{Tr}\left(\beta \wedge \beta^{*}\right)$. From this, one easily concludes that $\|\eta\|_{\omega} \leq 1$ by an appropriate choice of $\eta$, as well as $\left\|\Theta_{T_{X}, h}\right\|_{\omega, h} \leq 1$ and $\left\|\widetilde{\Theta}_{T_{X}, h}\right\|_{\omega, h} \leq 2$. By (2.33), we obtain for $n \geq 2$

$$
J \leq n^{3 / 2} \frac{\pi}{\sqrt{6}} \times 2 \frac{n^{n}-1}{n-1} \int_{X} \omega^{n}<\frac{4 \pi}{\sqrt{6}} n^{n+1 / 2} \int_{X} \omega^{n}
$$

where $\int_{X} \omega^{n}=\left(\sum d_{j}+1\right)^{n} \operatorname{deg}(X)$. On the other hand, the leading term $\int_{X} \eta^{n}$ equals $\left(\sum d_{j}-n-s-a-1\right)^{n} \operatorname{deg}(X)$ with $\operatorname{deg}(X)=d_{1} \ldots d_{s}$. By the bound (2.32) on the error term $\varepsilon_{k, r, n}$, we find that the leading coefficient of the growth of our spaces of sections is strictly controlled below by a multiple of

$$
\left(\sum d_{j}-n-s-a-1\right)^{n}-4 \pi\left(\frac{31}{90}\right)^{1 / 2} \frac{n^{n+1 / 2}}{\log k}\left(\sum d_{j}+1\right)^{n}
$$

if $k \geq e^{5 n-5}$. A sufficient condition for the existence of sections in (2.41) is thus

$$
k \geq \exp \left(7.38 n^{n+1 / 2}\left(\frac{\sum d_{j}+1}{\sum d_{j}-n-s-a-1}\right)^{n}\right) .
$$

This is good in view of the fact that we can cover arbitrary smooth complete intersections of general type. On the other hand, even when the degrees $d_{j}$ tend to $+\infty$, we still get a large lower bound $k \sim \exp \left(7.38 n^{n+1 / 2}\right)$ on the order of jets, and this is far from being optimal: Diverio [Div09] has shown e.g. that one can take $k=n$ for smooth hypersurfaces of high degree. It is however not unlikely that one could improve estimate (2.42) with more careful choices of $\omega, h$.

\section{ON THE BASE LOCUS OF SECTIONS OF $k$-JET BUNDLES}

The final step required for a complete solution of the Green-Griffiths conjecture would be to calculate the base locus $B_{k} \subset X_{k}^{\mathrm{GG}}$ of the space of sections

$H^{0}\left(X_{k}^{\mathrm{GG}}, \mathcal{O}_{X_{k}^{\mathrm{GG}}}(m) \otimes \pi_{k}^{*} \mathcal{O}\left(-m \delta_{k} A\right)\right), \quad A$ ample on $X, \quad \delta_{k} \leq c \frac{\log k}{k}, \quad c \ll 1$, and to show that $Y_{k}=\pi_{k}\left(B_{k}\right)$ is a proper algebraic subvariety of $X$ for $k$ large, under the assumption that $K_{V}$ is big. This does not look completely hopeless, 
since the statistics of curvature in the Morse inequalities do involve currents for which the sets of poles depend only on the bigness of $K_{V}$ and therefore project onto a proper subvariety $S$ of $X$ (see the last step of the proof in section 2). It is not unreasonable to think that a further analysis of the asymptotic behavior of sections, e.g. through estimates of the Bergman kernel, might lead to such results.

Even if the required property of the base locus cannot be obtained directly, it would be enough, for a suitable irreducible analytic set $Z \subset X_{k}^{\mathrm{GG}}$ contained in the base locus at some stage, to construct non zero sections in

$$
H^{0}\left(Z, \mathcal{O}_{X_{k}^{\mathrm{GG}}}(m)_{\mid Z} \otimes \pi_{k}^{*} \mathcal{O}\left(-m \delta_{k} A\right)_{\mid Z}\right)
$$

whenever $\pi_{k}(Z)=X$, and then to proceed inductively to cut-down the base locus until one reaches some $Z^{\prime} \subset Z$ with $\pi_{k}\left(Z^{\prime}\right) \subsetneq X$. Hence we have to estimate the cohomology groups $H^{0}$ and $H^{q}$ not just on $X_{k}^{\mathrm{GG}}$, but also on all irreducible subvarieties $Z \subset X_{k}^{\mathrm{GG}}$ such that $\pi_{k}(Z)=X$. We are not able to do this in such a generality, but our method does provide interesting results in this direction.

(3.1) Theorem. Let $(X, V)$ be a compact directed $n$-dimensional manifold, let $r=\operatorname{rank} V$ and $F$ be a holomorphic line bundle on $X$. Fix an irreducible analytic set $Z_{k_{0}} \subset X_{k_{0}}^{\mathrm{GG}}$ or equivalently some $\mathbb{C}^{*}$-invariant set $Z_{k_{0}}^{\prime} \subset J^{k_{0}} V$, and assume that $\pi_{k_{0}}\left(Z_{k_{0}}\right)=X$. For $k \gg k_{0}$, denote by $Z_{k} \subset X_{k}^{\mathrm{GG}}$ the irreducible set corresponding to the inverse image of $Z_{k_{0}}^{\prime}$ by the canonical morphism $J^{k} V \rightarrow J^{k_{0}} V$. Let $h$ be an admissible metric on $V, h_{F}$ a metric with analytic singularities on $F$ and

$$
\begin{aligned}
L_{k} & =\mathcal{O}_{X_{k}^{\mathrm{GG}}}(1) \otimes \pi_{k}^{*} \mathcal{O}\left(\frac{1}{k r}\left(1+\frac{1}{2}+\ldots+\frac{1}{k}\right) F\right), \\
\eta & =\Theta_{K_{V}, \operatorname{det} h^{*}}+\Theta_{F, h_{F}}, \quad S=\operatorname{Sing}(\eta) .
\end{aligned}
$$

Then for $m \gg k \gg k_{0}$ and $p_{k}=\operatorname{dim} Z_{k}=\operatorname{dim} Z_{k_{0}}+\left(k-k_{0}\right) r$ we have

$$
\begin{aligned}
& h^{0}\left(Z_{k}, \mathcal{O}\left(L_{k}^{\otimes m}\right)_{\mid Z_{k}}\right) \\
& \quad \geq \frac{m^{p_{k}}}{p_{k} !} \frac{(\log k)^{n}}{n !} \operatorname{deg}_{X_{k}^{\mathrm{GG}} / X}\left(Z_{k}\right)\left(\int_{X(\eta, \leq 1) \backslash S} \eta^{n}-O\left((\log k)^{-1}\right)\right)-o\left(m^{p_{k}}\right)
\end{aligned}
$$

where $\operatorname{deg}_{X_{k}^{\mathrm{GG}} / X}\left(Z_{k}\right)=\operatorname{deg}_{X_{k_{0}}^{\mathrm{GG}} / X}\left(Z_{k_{0}}\right)\left(\frac{k_{0} !}{k !}\right)^{r}$ is the relative degree of $Z_{k}$ over $X$ with respect to the normalized weighted "Kähler metric" $\omega_{a, r, p}$ introduced in (1.10). 
We would also get similar upper and lower Morse bounds for the higher cohomology groups, provided that the sheaves $\mathcal{O}_{X_{k}^{\mathrm{GG}}}(m)$ are twisted by the appropriate multiplier ideal sheaves $\mathcal{J}_{k, m}$ already described. The main trouble to proceed further in the analysis of the base locus is that we have to take $k \gg k_{0}$ and that the $O(\ldots)$ and $o(\ldots)$ bounds depend on $Z_{k_{0}}$. Hence the newer sections can only be constructed for higher and higher orders $k$, without any indication that we can actually terminate the process somewhere, except possibly by some extremely delicate uniform estimates which seem at present beyond reach.

Proof. The technique is a minor variation of what has been done in section 2, hence we will only indicate the basic idea. Essentially the $k$-jet of $f$ is no longer completely random, its projection onto the first $k_{0}$ components $\left(\nabla^{j} f(0)\right)_{1 \leq j \leq k_{0}}$ is assigned to belong to some given analytic set $Z_{k_{0}}^{\prime} \subset J^{k_{0}} V$. This means that in the curvature formula (2.15)

$$
g_{k}(z, x, u)=\frac{i}{2 \pi} \sum_{1 \leq s \leq k} \frac{1}{s} x_{s} \sum_{i, j, \alpha, \beta} c_{i j \alpha \beta}(z) u_{s \alpha} \bar{u}_{s \beta} d z_{i} \wedge d \bar{z}_{j}
$$

only the sum $\sum_{k_{0}<s \leq k}$ is perfectly random. The partial sum $\sum_{1 \leq s \leq k_{0}}$ remains bounded, while the harmonic series diverges as log $k$. This implies that the "non randomness" of the initial terms perturbs the estimates merely by bounded quantities, and in the end, the expected value is still similar to (2.18), i.e.

$$
\mathbf{E}\left(g_{k}(z, \bullet, \bullet)\right)=\frac{1}{k r}\left(1+\frac{1}{2}+\ldots+\frac{1}{k}+O(1)\right)\left(\Theta_{K_{V}, \operatorname{det} h^{*}}+\Theta_{F, h_{F}}\right) .
$$

Once we are there, the calculation of standard deviation and the other estimates are just routine, and Theorem 3.1 follows again from Proposition 2.13 when we integrate the Morse integrals over $Z_{k}$ instead of the whole $k$-jet space $X_{k}^{\mathrm{GG}}$.

Another possibility to analyze the base locus is to study the restriction maps

$$
\rho_{k, m}(x): H^{0}\left(X, E_{k, m}^{\mathrm{GG}} V^{*} \otimes \mathcal{O}\left(-m \delta_{k} A\right)\right) \rightarrow\left(E_{k, m}^{\mathrm{GG}} V^{*} \otimes \mathcal{O}\left(-m \delta_{k} A\right)\right)_{x}
$$

at generic points $x \in X$. If $\rho_{k, m}(x)$ can be shown to be surjective at a generic point, then a fortiori the projection $Y_{k}=\pi_{k}\left(B_{k}\right)$ of the base locus does not contain $x$ and so $Y_{k}$ is a proper algebraic subvariety of $X$. Now, proving the surjectivity of $\rho_{k, m}(x)$ could be done by proving the vanishing of the $H^{1}$ group of our sheaf twisted by the maximal ideal $\mathfrak{m}_{X, x}$. We cannot exactly reach such a 
precise vanishing result, but Morse inequalities can be used to show that the $H^{1}$ groups do not grow too fast.

In fact assume that $A$ is an ample $\mathbb{Q}$-divisor on $X$ which is chosen so small that $K_{V} \otimes \mathcal{O}(-A)$ is still big. By our estimates, we can then take $\delta_{k}=\frac{1}{k r}\left(1+\frac{1}{2}+\ldots+\frac{1}{k}\right)$. Pick a very ample divisor $G$ on $X$ and $n$ pencils of sections $\sigma_{j, t} \in H^{0}(X, \mathcal{O}(G))$, $1 \leq j \leq n, t \in \mathbb{P}_{\mathbb{C}}^{1}$, such that the divisors $\sigma_{j, t_{j}}(z)=0$ intersect transversally at isolated points for generic choices of the parameters $t_{j} \in \mathbb{P}_{\mathbb{C}}^{1}$. We select an admissible metric $h$ on $V$ which provides a strictly positive curvature current on $K_{V} \otimes \mathcal{O}(-A)$ and multiply it by the additional weight factor $\left(e^{\varphi}\right)^{1 / r m \delta_{k}}$ where

$$
\varphi(z)=\log \sum_{1 \leq j \leq n} \prod_{t \in T_{j}}\left|\sigma_{j, t}(z)\right|_{h_{G}}^{2 n}
$$

and $T_{j} \subset \mathbb{P}_{\mathbb{C}}^{1}$ are generic finite subsets of given cardinality $N$. The multiplier ideal sheaf of $\varphi$ is precisely equal to the ideal $\mathcal{I}_{E}$ of germs of functions vanishing on a certain 0-dimensional set $E=\left\{x_{1}, \ldots, x_{s}\right\} \subset X$ of cardinality $s=N^{n} G^{n}$. Also the resulting curvature form

$$
\eta=\Theta_{K_{V}, \operatorname{det} h^{*}}-\Theta_{A, h_{A}}+\frac{1}{m \delta_{k}} d d^{c} \varphi \geq \Theta_{K_{V}, \operatorname{det} h^{*}}-\Theta_{A, h_{A}}-\frac{N}{m \delta_{k}} \Theta_{G, h_{G}}
$$

can be made to be strictly positive as a current provided that $N \sim c m \delta_{k}$ with $c \ll 1$. Then the corresponding multiplier ideal sheaf of the induced hermitian metric on

$$
\mathcal{O}_{X_{k}^{\mathrm{GG}}}(m) \otimes \pi_{k}^{*} \mathcal{O}\left(-m \delta_{k} A\right)
$$

is the original multiplier sheaf $\mathcal{J}_{k, m}$ twisted by $\pi_{k}^{*} \mathcal{I}_{E}$ above $x_{j}$, provided that the $x_{j}$ lie outside of $\operatorname{Sing}(V)$ and outside of the projection of the support $V\left(\mathcal{J}_{k, m}\right)$. Consider the exact sequence

$$
\begin{aligned}
0 \longrightarrow \mathcal{O}_{X_{k}^{\mathrm{GG}}}(m) \otimes \pi_{k}^{*} \mathcal{O}\left(-m \delta_{k} A\right) \otimes \mathcal{J}_{k, m} \otimes \pi_{k}^{*} \mathcal{I}_{E} \\
\longrightarrow \mathcal{O}_{X_{k}^{\mathrm{GG}}}(m) \otimes \pi_{k}^{*} \mathcal{O}\left(-m \delta_{k} A\right) \otimes \mathcal{J}_{k, m} \\
\longrightarrow \mathcal{O}_{X_{k}^{\mathrm{GG}}}(m) \otimes \pi_{k}^{*} \mathcal{O}\left(-m \delta_{k} A\right) \otimes \mathcal{J}_{k, m} \otimes \pi_{k}^{*}\left(\mathcal{O}_{X} / \mathcal{I}_{E}\right) \longrightarrow 0 .
\end{aligned}
$$

Its cohomology exact sequence yields an "almost surjective arrow"

$$
H^{0}\left(\mathcal{O}_{X_{k}^{\mathrm{GG}}}(m) \otimes \pi_{k}^{*} \mathcal{O}\left(-m \delta_{k} A\right) \otimes \mathcal{J}_{k, m}\right) \longrightarrow \bigoplus_{1 \leq j \leq s}\left(E_{k, m}^{\mathrm{GG}} V^{*} \otimes \mathcal{O}\left(-m \delta_{k} A\right)\right)_{x_{j}},
$$

namely the image contains the kernel of the map

$$
\bigoplus_{1 \leq j \leq s}\left(E_{k, m}^{\mathrm{GG}} V^{*} \otimes \mathcal{O}\left(-m \delta_{k} A\right)\right)_{x_{j}} \longrightarrow H^{1}\left(\mathcal{O}_{X_{k}^{\mathrm{GG}}}(m) \otimes \pi_{k}^{*} \mathcal{O}\left(-m \delta_{k} A\right) \otimes \mathcal{J}_{k, m} \otimes \pi_{k}^{*} \mathcal{I}_{E}\right) .
$$


Now, we have a Morse upper bound

$h^{1}\left(\mathcal{O}_{X_{k}^{\mathrm{GG}}}(m) \otimes \pi_{k}^{*} \mathcal{O}\left(-m \delta_{k} A\right) \otimes \mathcal{J}_{k, m} \otimes \pi_{k}^{*} \mathcal{I}_{E}\right) \leq \frac{m^{n+k r-1}}{(n+k r-1) !} \frac{(\log k)^{n}}{n !(k !)^{r}} O\left((\log k)^{-1}\right)$

since the 1-index integral $\int_{X(\eta, 1)} h^{n}$ is identically zero. At the same time we have $s=N^{n} G^{n} \sim c^{\prime} m^{n}(\log k)^{n} / k^{n}$, and it follows that

$\operatorname{dim} \bigoplus_{1 \leq j \leq s}\left(E_{k, m}^{\mathrm{GG}} V^{*} \otimes \mathcal{O}\left(-m \delta_{k} A\right)\right)_{x_{j}} \sim s \frac{m^{k r-1}}{(k r-1) !(k !)^{r}} \sim \frac{c^{\prime} m^{n+k r-1}}{(k r-1) !(k !)^{r}} \frac{(\log k)^{n}}{k^{n}}$.

By selecting a suitable point $x_{j}$ and by using a trivial lower semi-continuity argument, we get the desired almost surjectivity.

(3.3) Corollary. If $A$ is an ample $\mathbb{Q}$-divisor on $X$ such that $K_{V} \otimes \mathcal{O}(-A)$ is big and $\delta_{k}=\frac{1}{k r}\left(1+\frac{1}{2}+\ldots+\frac{1}{k}\right), r=\operatorname{rank} V$, the restriction map

$$
\rho_{k, m}(x): H^{0}\left(X, E_{k, m}^{\mathrm{GG}} V^{*} \otimes \mathcal{O}\left(-m \delta_{k} A\right)\right) \rightarrow\left(E_{k, m}^{\mathrm{GG}} V^{*} \otimes \mathcal{O}\left(-m \delta_{k} A\right)\right)_{x}
$$

has an image of dimension larger than $\left(1-O\left((\log k)^{-1}\right)\right) \operatorname{dim} E_{k, m}^{\mathrm{GG}} V^{*}$ at a generic point $x \in X$ for $m \gg k \gg 1$.

Such a result puts an upper bound on the vanishing order that a generic section may have on $X_{k}^{\mathrm{GG}}$ above a generic point of $X$. Our hope is that one can then completely "eliminate" the base locus by taking vertical derivatives along the fibers of $J^{k} V \rightarrow X$; those derivations will necessarily have some poles $\mathcal{O}(p A)$ which we hope to get cancelled by the negative powers $\mathcal{O}\left(-m \delta_{k} A\right)$. This strategy first devised by [Siu02, Siu04] has indeed been successful in some cases for the study of generic algebraic degeneracy (e.g. for hypersurfaces of very large degree in $\left.\mathbb{P}_{\mathbb{C}}^{n+1}\right)$. This would work rather easily if the rough error term $O\left((\log k)^{-1}\right)$ could be replaced e.g. by $O\left(m^{-\varepsilon_{k}}\right)$ in Corollary (3.3), but this is maybe too much to ask for.

We finally discuss yet another approach. For this we have to introduce invariant jet differentials along the lines of [Dem95]. In fact, to any directed manifold $(X, V)$ one can associate its tower of Semple $k$-jet spaces, which is a sequence of directed pairs $\left(X_{k}, V_{k}\right)$ starting with $\left(X_{0}, V_{0}\right)=(X, V)$, together with morhisms $\widetilde{\pi}_{k}:\left(X_{k}, V_{k}\right) \rightarrow\left(X_{k-1}, V_{k-1}\right)$. These spaces are constructed inductively by putting $X_{k}=P\left(V_{k-1}\right)$ and $V_{k}=\left(\widetilde{\pi}_{k}\right)_{*}^{-1}\left(\mathcal{O}_{X_{k}}(-1)\right)$ where

$$
\mathcal{O}_{X_{k}}(-1) \subset\left(\widetilde{\pi}_{k}\right)^{*} V_{k-1} \subset\left(\widetilde{\pi}_{k}\right)^{*} T_{X_{k-1}}
$$


is the tautological subbundle (cf. [Dem95]). In the case where $V$ is not a subbundle, we can first construct the absolute tower $\left(\bar{X}_{k}, \bar{V}_{k}\right)$ by starting from $\bar{V}_{0}=T_{X}$, and then take $X_{k}$ to be the closure in $\bar{X}_{k}$ of the $k$-step $X_{k}^{\prime}$ of the relative tower $\left(X_{k}^{\prime}, V_{k}^{\prime}\right)$ constructed over the dense Zariski open set $X^{\prime}=X \backslash \operatorname{Sing}(V)$. In this way, the tower $\left(X_{k}, V_{k}\right)$ is at least birationally well defined - in such a birational context we can even assume that $X_{k}$ is smooth after performing a suitable modification at each stage. Even if we start with $V=T_{X}$ (or an integrable subbundle $V \subset T_{X}$ ), the $k$-jet lifting $V_{k}$ will not be integrable in general, the only exception being when $\operatorname{rank} V_{k}=\operatorname{rank} V=1$. Now, if

$$
\pi_{k, 0}=\tilde{\pi}_{k} \circ \ldots \circ \widetilde{\pi}_{1}: X_{k} \rightarrow X_{0}=X,
$$

it is shown in [Dem95] that the direct image sheaf

$$
\pi_{k, 0} \mathcal{O}_{X_{k}}(m):=E_{k, m} V^{*} \subset E_{k, m}^{\mathrm{GG}} V^{*}
$$

consists of algebraic differential operators $P\left(f_{j \leq k}^{(j)}\right)$ which satisfy the invariance property

$$
P\left((f \circ \varphi)_{j \leq k}^{(j)}\right)=\left(\varphi^{\prime}\right)^{m} P\left(f_{j \leq k}^{(j)}\right) \circ \varphi
$$

when $\varphi \in \mathbb{G}_{k}$ is in the group of $k$-jets of biholomorphisms $\varphi:(\mathbb{C}, 0) \rightarrow(\mathbb{C}, 0)$. Since we already assume $\mathbb{C}^{*}$ invariance, it is enough to require invariance by the nilpotent subgroup $\mathbb{G}_{k}^{\prime} \subset \mathbb{G}_{k}$ of $k$-jets tangent to identity. The group $\mathbb{G}_{k}^{\prime}$ is a semi-direct product of additive groups $(\mathbb{C},+)$ consisting of biholomorphisms $\tau_{j, a}: t \mapsto t+a t^{j}+O\left(t^{j+1}\right), 2 \leq j \leq k, a \in \mathbb{C}$. In this tower, the biholomorphisms $\tau_{k, a}$ actually generate a normal subgroup of $\mathbb{G}_{k}^{\prime}$, and we have $\mathbb{G}_{k}^{\prime} /\left\{\tau_{k, a}\right\} \simeq \mathbb{G}_{k-1}^{\prime}$. Now, assume that we have found a section

$$
P \in H^{0}\left(X, E_{k, m}^{\mathrm{GG}} V^{*} \otimes \mathcal{O}\left(-m \delta_{k} A\right)\right)
$$

for some ample $\mathbb{Q}$-divisor $A$ on $A$. Then we have an expansion

$$
P_{a}\left(f_{j \leq k}^{(j)}\right):=P\left(\left(f \circ \tau_{k, a}\right)_{j \leq k}^{(j)}\right)=\sum_{0 \leq s \leq m / k} a^{s} P_{s}\left(f_{j \leq k}^{(j)}\right)
$$

and the highest non zero term $P_{s}$ is $\left\{\tau_{k, a}\right\}$-invariant of weighted degree $m-(k-1) s$; this comes from the fact that the homothety $h_{\lambda}(t)=\lambda t$ satisfies

$$
\tau_{k, a} \circ h_{\lambda}=h_{\lambda} \circ \tau_{k, a \lambda^{k-1}}
$$

Then it makes sense to look at the action of $\left\{\tau_{k-1, a}\right\}$ on $P_{s}$, and proceeding inductively we reach a non zero $\mathbb{G}_{k}^{\prime}$-invariant (and thus $\mathbb{G}_{k}$-invariant) polynomial

$$
Q \in H^{0}\left(X, E_{k, m^{\prime}} V^{*} \otimes \mathcal{O}\left(-m \delta_{k} A\right)\right)
$$


of degree $m^{\prime} \leq m$ (and possibly of order $k^{\prime} \leq k$ but we can still consider it to be of order $k$ ). By raising $Q$ to some power $p$ and using the $\mathbb{Q}$-ampleness of $A$, we obtain a genuine integral section

$$
Q^{p} \sigma_{A}^{p\left(m-m^{\prime}\right) \delta_{k}} \in H^{0}\left(X, E_{k, p m^{\prime}} V^{*} \otimes \mathcal{O}\left(-p m^{\prime} \delta_{k} A\right)\right) .
$$

(3.4) Corollary. Let $(X, V)$ be a projective directed manifold such that $K_{V}$ is big, and $A$ an ample $\mathbb{Q}$-divisor on $X$ such that $K_{V} \otimes \mathcal{O}(-A)$ is still big. Then, if we put $\delta_{k}=\frac{1}{k r}\left(1+\frac{1}{2}+\ldots+\frac{1}{k}\right), r=\operatorname{rank} V$, the space of global invariant jet differentials

$$
H^{0}\left(X, E_{k, m} V^{*} \otimes \mathcal{O}\left(-m \delta_{k} A\right)\right)
$$

has (many) non zero sections for $m \gg k \gg 1$.

If we have a directed projective variety $(X, V)$ with $K_{V}$ big, we conclude that there exists $k \geq 1$ and a proper analytic set $Z \subset X_{k}$ such that all entire curves have the image of their $k$-jet $f_{[k]}(\mathbb{C})$ contained in $Z$. Let $Z^{\prime}$ be an irreducible component of $Z$ such that $\pi_{k, 0}\left(Z^{\prime}\right)=X$ (if $\pi_{k, 0}\left(Z^{\prime}\right) \subsetneq X$ there is nothing more to do). Consider the linear subspace $V^{\prime}=\overline{T_{Z^{\prime}} \backslash Z^{\prime \prime}} \cap V_{k}$ where $Z^{\prime \prime} \subset Z^{\prime}$ is chosen such that $Z^{\prime} \backslash Z^{\prime \prime}$ is non singular and the intersection $T_{Z^{\prime} \backslash Z^{\prime \prime}} \cap V_{k}$ is a subbundle of $T_{Z^{\prime} \backslash Z^{\prime \prime}}$. If $f_{[k]}(\mathbb{C})$ is not contained identically in $Z^{\prime \prime}$, then the curve $g=f_{[k]}$ is tangent to $\left(Z^{\prime}, V^{\prime}\right)$. On the other hand, if $f_{[k]}(\mathbb{C}) \subset Z^{\prime \prime}$ we can replace $Z^{\prime}$ by $Z^{\prime \prime}$ and argue inductively on $\operatorname{dim} Z^{\prime}$. What we have gained here is that we have replaced the initial directed space $(X, V)$ with another one $\left(Z^{\prime}, V^{\prime}\right)$ such that $\operatorname{rank} V^{\prime}<\operatorname{rank} V$, and we can try to $\operatorname{argue}$ by induction on $r=\operatorname{rank} V$.

Observe that the generalized Green-Griffiths conjecture is indeed trivial for $r=1$ (assuming $K_{V}=\mathcal{O}\left(V^{*}\right)$ big) : in fact we get in this case a non zero section $P \in H^{0}\left(X, V^{* \otimes k} \otimes \mathcal{O}(-A)\right)$ for some $k \gg 1$ and so $P(f) \cdot\left(f^{\prime}\right)^{k}$ must vanish for every entire curve $f:\left(\mathbb{C}, T_{\mathbb{C}}\right) \rightarrow(X, V)$. Therefore

$$
f(\mathbb{C}) \subset Y:=\{P(z)=0\} \subsetneq X .
$$

The main difficulty in this inductive approach is that when we start with $(X, V)$ with $K_{V}$ big, it seems to be very hard to say anything about $K_{V^{\prime}}$ on $\left(Z^{\prime}, V^{\prime}\right)$. Especially, the singularities of $Z^{\prime}$ and $V^{\prime}$ do not seem to be under control. The only hope would be to have enough control on the sections cutting out $Z^{\prime}$, and this requires anyway to understand much more precisely the behavior and vanishing order of generic sections $P \in H^{0}\left(X, E_{k, m} V^{*} \otimes \mathcal{O}\left(-m \delta_{k} A\right)\right)$. One could try in 
this context to take $A$ to approach the positive part in the Zariski decomposition of $K_{V}$, in such a way that the sections $P$ do not have much space to move around statistically.

\section{REFERENCES}

[Ber10] Bérczi, G.: Thom polynomials and the Green-Griffiths conjecture. Manuscript Math. Institute Oxford, May 2010.

[Blo26] Bloch, A.: Sur les systèmes de fonctions uniformes satisfaisant à l'équation d'une variété algébrique dont l'irrégularité dépasse la dimension. J. de Math., 5 (1926), 19-66.

[Bog79] Bogomolov, F.A.: Holomorphic tensors and vector bundles on projective varieties. Math. USSR Izvestija 13/3 (1979), 499-555.

[Bon93] Bonavero, L.: Inégalités de morse holomorphes singulières. Acad. Sci. Paris Sér. I Math. 317 (1993), 1163-1166, and: J. Geom. Anal. 8 (1998), 409-425.

[Bro78] Brody, R.: Compact manifolds and hyperbolicity. Trans. Amer. Math. Soc. 235 (1978), 213-219.

[BG77] Brody, R., Green, M.: A family of smooth hyperbolic surfaces in $\mathbb{P}^{3}$. Duke Math. J. 44 (1977), 873-874.

[Cle86] Clemens, H.: Curves on generic hypersurfaces. Ann. Sci. Éc. Norm. Sup. 19 (1986) 629-636, Erratum: Ann. Sci. Éc. Norm. Sup. 20 (1987) 281.

[CG76] Cowen, M., Griffiths, P.: Holomorphic curves and metrics of negative curvature. J. Analyse Math. 29 (1976), 93-153.

[Dem85] Demailly, J.-P.: Champs magnétiques et inégalités de Morse pour la d" -cohomologie. Ann. Inst. Fourier (Grenoble), 35 (1985), 189-229.

[Dem95] Demailly, J.-P.: Algebraic criteria for Kobayashi hyperbolic projective varieties and jet differentials. AMS Summer School on Algebraic Geometry, Santa Cruz 1995, Proc. Symposia in Pure Math., ed. by J. Kollár and R. Lazarsfeld, 76p.

[Dem97] Demailly, J.-P.: Variétés hyperboliques et équations différentielles algébriques. Gaz. Math. 73 (juillet 1997), 3-23.

[Dem10a] Demailly, J.-P.: Holomorphic Morse inequalities and asymptotic cohomology groups: a tribute to Bernhard Riemann. arXiv: math.CV/1003.5067.

[Dem10b] Demailly, J.-P.: A converse to the Andreotti-Grauert theorem. Manuscript Inst. Fourier Grenoble, October 2010.

[DEG00] Demailly, J.-P., El Goul, J.: Hyperbolicity of generic surfaces of high degree in projective 3-space. Amer. J. Math. 122 (2000), 515-546.

[Div09] Diverio, S.: Existence of global invariant jet differentials on projective hypersurfaces of high degree. Math. Ann. 344 (2009), 293-315.

[DMR10] Diverio, S., Merker, J., Rousseau, E.: Effective algebraic degeneracy. Invent. Math. 180 (2010), 161-223.

[DT10] Diverio, S., Trapani, S.: A remark on the codimension of the Green-Griffiths locus of generic projective hypersurfaces of high degree. To appear in J. Reine Angew. Math. 
[Fuj94] Fujita, T.: Approximating Zariski decomposition of big line bundles. Kodai Math. J. 17 (1994), 1-3.

[GG79] Green, M., Griffiths, P.: Two applications of algebraic geometry to entire holomorphic mappings. The Chern Symposium 1979, Proc. Internal. Sympos. Berkeley, CA, 1979, Springer-Verlag, New York (1980), 41-74.

[Kob70] Kobayashi, S.: Hyperbolic manifolds and holomorphic mappings. Marcel Dekker, New York (1970).

[Kob76] Kobayashi, S.: Intrinsic distances, measures and geometric function theory. Bull. Amer. Math. Soc. 82 (1976), 357-416.

[KobO75] Kobayashi, S., Ochiai, T.: Meromorphic mappings into compact complex spaces of general type. Invent. Math. 31 (1975), 7-16.

[Lang86] Lang, S.: Hyperbolic and Diophantine analysis. Bull. Amer. Math. Soc. 14 (1986) 159-205.

[Lang87] Lang, S.: Introduction to complex hyperbolic spaces. Springer-Verlag, New York (1987).

[McQ96] McQuillan, M.: A new proof of the Bloch conjecture. J. Alg. Geom. 5 (1996), 107-117.

[McQ98] McQuillan, M.: Diophantine approximation and foliations. Inst. Hautes Études Sci. Publ. Math. 87 (1998), 121-174

[McQ99] McQuillan, M.: Holomorphic curves on hyperplane sections of 3-folds. Geom. Funct. Anal. 9 (1999), 370-392.

[Mer08] Merker, J.: Jets de Demailly-Semple dordres 4 et 5 en dimension 2. arXiv: math.AG/0710.2393, Int. J. Contemp. Math. Sciences 3 (2008) 861-933.

[Mer09] Merker, J.: Low pole order frames on vertical jets of the universal hypersurface. arXiv: math.AG/0805.3987, Ann. Inst. Fourier (Grenoble) 59 (2009) 1077-1104.

[Mer10] Merker, J.: Complex projective hypersurfaces of general type: toward a conjecture of Green and Griffiths. Manuscript Éc. Norm. Sup. Paris, May 2010, arXiv: math.AG/1005.0405.

[Pau08] Păun, M.: Vector fields on the total space of hypersurfaces in the projective space and hyperbolicity. Math. Ann. 340 (2008) 875-892.

[Rou06a] Rousseau, E.: Étude des jets de Demailly-Semple en dimension 3. Ann. Inst. Fourier (Grenoble) 56 (2006) 397-421.

[Rou06b] Rousseau, E.: Équations différentielles sur les hypersurfaces de $\mathbb{P}^{4}$. J. Math. Pures Appl. 86 (2006) 322-341.

[Rou07] Rousseau, E.: Weak analytic hyperbolicity of generic hypersurfaces of high degree in $\mathbb{P}^{4}$. Annales Fac. Sci. Toulouse 16 (2007), 369-383.

[Siu97] Siu, Y.T.: A proof of the general schwarz lemma using the logarithmic derivative lemma. Communication personnelle, avril 1997.

[Siu02] Siu, Y.T.: Some recent transcendental techniques in algebraic and complex geometry. Proceedings of the International Congress of Mathematicians, Vol. I (Beijing, 2002), Higher Ed. Press, Beijing, 2002, 439-448.

[Siu04] Siu, Y.T.: Hyperbolicity in complex geometry. The legacy of Niels Henrik Abel, Springer, Berlin, 2004, 543-566. 
Holomorphic Morse Inequalities and The Green-Griffiths-Lang... 1207

[SY96a] Siu, Y.T., Yeung, S.K.: Hyperbolicity of the complement of a generic smooth curve of high degree in the complex projective plane. Invent. Math. 124 (1996), 573-618.

[SY96b] Siu, Y.T., Yeung, S.K.: Defects for ample divisors of Abelian varieties, Schwarz lemma and hyperbolic surfaces of low degree. Preprint (prépublication, automne 1996).

[Tra95] Trapani, S.: Numerical criteria for the positivity of the difference of ample divisors. Math. Z. 219 (1995), 387-401.

[Voi96] Voisin, C.: On a conjecture of Clemens on rational curves on hypersurfaces. J. Diff. Geom. 44 (1996), 200-213. Correction: J. Diff. Geom. 49 (1998), 601-611.

Jean-Pierre Demailly

Université de Grenoble I, Département de Mathématiques

Institut Fourier, 38402 Saint-Martin d'Hères, France

E-mail: demailly@fourier.ujf-grenoble.fr 